Groups Geom. Dyn. 4 (2010), 275-307

DOI $10.4171 / \mathrm{GGD} / 84$
Groups, Geometry, and Dynamics

(C) European Mathematical Society

\title{
On right-angled Artin groups without surface subgroups
}

\author{
Sang-hyun Kim
}

\begin{abstract}
We study the class $\mathcal{N}$ of graphs, the right-angled Artin groups defined on which do not contain closed hyperbolic surface subgroups. We prove that a presumably smaller class $\mathcal{N}^{\prime}$ is closed under amalgamating along complete subgraphs, and also under adding bisimplicial edges. It follows that chordal graphs and chordal bipartite graphs belong to $\mathcal{N}^{\prime}$.
\end{abstract}

Mathematics Subject Classification (2010). Primary 20F36, 20F65; Secondary 05C25.

Keywords. Right-angled Artin group, graph group, surface group, label-reading map.

\section{Introduction}

In this paper, all graphs will be finite and will not have loops or multi-edges unless specified otherwise. For a graph $\Gamma$, let $V(\Gamma)$ and $E(\Gamma)$ denote the vertex set and the edge set of $\Gamma$, respectively. The right-angled Artin group with the underlying graph $\Gamma$ is the group presentation

$$
A(\Gamma)=\langle V(\Gamma)|[u, v]=1 \text { for }\{u, v\} \in E(\Gamma)\rangle .
$$

Also known as graph groups or partially commutative groups, right-angled Artin groups possess various group theoretic properties. One of the most fundamental results is that, two right-angled Artin groups are isomorphic if and only if their underlying graphs are isomorphic [20], [10]. Right-angled Artin groups are linear [19], [18], [7], biorderable [12], [6], and acting on finite-dimensional CAT(0) cube complexes freely and cocompactly [4], [23], [25]. Any subgroup of a right-angled Artin group surjects onto $\mathbb{Z}[17]$.

The complement graph of a graph $\Gamma$ is the graph $\bar{\Gamma}$, defined by $V(\bar{\Gamma})=V(\Gamma)$ and $E(\bar{\Gamma})=\{\{u, v\} \mid\{u, v\} \notin E(\Gamma)\}$. For a subset $S$ of $V(\Gamma)$, the induced subgraph $\Gamma_{S}$ of $\Gamma$ on $S$ is the maximal subgraph of $\Gamma$ with the vertex set $S$. We also write $\Gamma_{S} \leq \Gamma$. Note that $V\left(\Gamma_{S}\right)=S$ and $E\left(\Gamma_{S}\right)=\{\{u, v\} \mid u, v \in S$ and $\{u, v\} \in E(\Gamma)\}$. If $\Lambda$ is another graph, an induced $\Lambda$ in $\Gamma$ is an induced subgraph of $\Gamma$, which is isomorphic to $\Lambda$. An elementary fact is, if $\Gamma$ contains an induced $\Lambda$, then $A(\Lambda)$ embeds into $A(\Gamma)$. A complete graph $K_{n}$ is a graph with $n$ vertices such that every pair of distinct vertices 
are joined by an edge. For convention, $K_{0}=\varnothing$ is considered also as a complete graph. $C_{n}$ and $P_{n}$ denote the cycle and the path with $n$ vertices, respectively. In particular, $P_{n}$ is obtained by removing an edge in $C_{n} . C_{3}$ is also called a triangle. A graph is chordal if the graph does not contain any induced cycle of length at least 4 . Graph theoretic characterizations of $\Gamma$ determine several group theoretic properties of $A(\Gamma)$ :

- $A(\Gamma)$ is coherent (i.e., every finitely generated subgroup is finitely presented), if and only if $\Gamma$ is chordal [9], if and only if $A(\Gamma)$ has a free commutator subgroup [27].

- $A(\Gamma)$ is subgroup separable (i.e., every finitely generated subgroup is closed in the profinite topology), if and only if $\Gamma$ does not contain an induced $C_{4}$ or an induced $P_{4}$ [24], which happens exactly when every subgroup of $A(\Gamma)$ is also a right-angled Artin group [11].

- $A(\Gamma)$ is virtually a 3-manifold group, if and only if each connected component of $\Gamma$ is a tree or a triangle [9], [15].

In this paper, a surface will mean a compact, oriented 2-manifold. A closed (compact, respectively) hyperbolic surface group will mean the fundamental group of a closed (compact, respectively) hyperbolic surface. Finding sufficient and necessary conditions for a given group to contain a closed hyperbolic surface group is an important question motivated by 3-manifold theory. In this article, we consider this question in the case of right-angled Artin groups. Namely, we investigate

$$
\mathcal{N}=\{\Gamma \mid A(\Gamma) \text { does not contain a closed hyperbolic surface group }\} .
$$

$A(\Gamma)$ is known to contain a closed hyperbolic surface group if $\Gamma$ has an induced $C_{n}(n \geq 5)$ [27] or an induced $\overline{C_{n}}(n \geq 5)$ [22] (proved by the author). That is, $C_{n}$ and $\overline{C_{n}}$ are not in $\mathcal{N}$ for $n \geq 5$. The classification of the graphs in $\mathcal{N}$ with at most 8 vertices is given in [5]. A complete graph amalgamation of two graphs is the union of the two graphs such that their intersection is complete; in particular, those two graphs will be induced subgraphs of the union. A key stumbling block for the (graph theoretic) characterization of $\mathcal{N}$ is the following conjecture.

Conjecture 1.1. $\mathcal{N}$ is closed under complete graph amalgamation.

For a graph $\Gamma$, a cube complex $X_{\Gamma}$ is inductively defined as follows.

(i) $X_{\Gamma}^{(0)}$ is a single vertex.

(ii) Suppose that $X_{\Gamma}^{(k-1)}$ is constructed, so that each complete subgraph of $\Gamma$ with $i$ vertices $(i<k)$ corresponds to an $i$-torus. Let $K$ be a complete subgraph of $\Gamma$ with $k$ vertices. Glue a unit $k$-cube to $X_{\Gamma}^{(k-1)}$ so that each pair of parallel faces is glued to each $(k-1)$-torus corresponding to the complete subgraph of $K$ with $k-1$ vertices. 
$X_{\Gamma}$, called the Salvetti complex of $A(\Gamma)$, is a locally CAT $(0)$ cube complex on which $A(\Gamma)$ acts freely and cocompactly. In particular, $X_{\Gamma}$ is a $K(A(\Gamma), 1)$-space [3].

Suppose that $\Gamma=\Gamma_{1} \cup \Gamma_{2}$, such that $K=\Gamma_{1} \cap \Gamma_{2}$ is complete and $\Gamma \notin \mathcal{N}$. One can find a closed hyperbolic surface $S$, and a $\pi_{1}$-injective map $f: S \rightarrow X_{\Gamma}$. Since $A(\Gamma)$ is the amalgamated free product of $A\left(\Gamma_{1}\right)$ and $A\left(\Gamma_{2}\right)$ along a free abelian subgroup $A(K)$, a transversality argument shows that there exists a compact hyperbolic surface $S_{1} \subseteq S$ and a $\pi_{1}$-injective map $g: S_{1} \rightarrow X_{\Gamma_{i}}$ such that $g\left(\partial S_{1}\right) \subseteq X_{K}$, where $i=1$ or 2 . In order to approach Conjecture 1.1 , it is natural to consider a relative embedding of a compact hyperbolic surface group into $A(\Gamma)$ as follows.

Definition 1.2. Let $\Gamma$ be a graph and $S$ be a compact hyperbolic surface. An embedding $\phi: \pi_{1}(S) \rightarrow A(\Gamma)$ is called a relative embedding if $\phi=f_{*}$ for some $\pi_{1}$-injective map $f: S \rightarrow X_{\Gamma}$ satisfying the following:

for each boundary component $\partial_{i} S$ of $S$, there exists a complete subgraph $K \leq \Gamma$ such that $f\left(\partial_{i} S\right) \subseteq X_{K}$.

Define $\mathcal{N}^{\prime}$ to be the class of graphs $\Gamma$ such that $A(\Gamma)$ does not allow a relative embedding of a compact hyperbolic surface group. It is vacuously true that $\mathcal{N}^{\prime} \subseteq \mathcal{N}$. Also, the paragraph preceding Definition 1.2 has proved the following.

Lemma 1.3. If $\Gamma \notin \mathcal{N}$ and $\Gamma$ is a complete graph amalgamation of $\Gamma_{1}$ and $\Gamma_{2}$, then $\Gamma_{i} \notin \mathcal{N}^{\prime}$ for $i=1$ or 2 .

For a compact surface $S$ and a set $V$, a $V$-dissection on $S$ is a pair $(\mathscr{H}, \lambda)$ such that $\mathscr{H}$ is a set of transversely oriented simple closed curves and properly embedded arcs on $S$, and $\lambda: \mathscr{H} \rightarrow V$ [6]. For each $\gamma \in \mathscr{H}, \lambda(\gamma)$ is called the label of $\gamma$. Note that our definition allows curves or arcs of the same label to intersect, while the definition in [6] does not. Let $\Gamma$ be a graph and $(\mathscr{H}, \lambda)$ be a $V(\Gamma)$-dissection. Suppose that for any $\alpha$ and $\beta$ in $\mathscr{H}, \alpha \cap \beta \neq \varnothing$ only if $\lambda(\alpha)$ and $\lambda(\beta)$ are equal or adjacent in $\Gamma$. Then $(\mathscr{H}, \lambda)$ determines a map $\phi: \pi_{1}(S) \rightarrow A(\Gamma)$. $\phi$ maps the equivalence class of a based, oriented loop $\alpha \subseteq S$ onto the word in $A(\Gamma)$, obtained by reading off the labels of the curves and the arcs in $\mathscr{H}$ that $\alpha$ intersects, and recording the order and the transverse orientations of the intersections. That is, when $\alpha$ crosses $\gamma \in \mathcal{H}$, one records $\lambda(\gamma)$ or $\lambda(\gamma)^{-1}$, according to whether the orientation of $\alpha$ matches the transverse orientation of $\gamma$. This map $\phi: \pi_{1}(S) \rightarrow A(\Gamma)$ is called the label-reading map with respect to (or, induced by) $(\mathscr{H}, \lambda)$, and $(\mathscr{H}, \lambda)$ is called a label-reading pair with the underlying graph $\Gamma$. In [6], it is shown that any map $\pi_{1}(S) \rightarrow A(\Gamma)$ can be realized as a label-reading map. By studying this label-reading pair, we will prove that a relative embedding of a compact hyperbolic surface group into $A(\Gamma)$ can be promoted to an embedding of a closed hyperbolic surface group into $A\left(\Gamma^{*}\right)$, for some graph $\Gamma^{*}$ which is strictly larger than $\Gamma$ (Lemma 3.10). This plays a crucial role in the proof of the following theorem.

Theorem 3.12. $\mathcal{N}^{\prime}$ is closed under complete graph amalgamation. 
$K_{n} \in \mathcal{N}^{\prime}$, since $A\left(K_{n}\right) \cong \mathbb{Z}^{n}$ does not contain any non-abelian free group. A classical result of Dirac shows that any chordal graph can be constructed by taking complete graph amalgamations successively, starting from complete graphs [8], [14]. So, we have:

Corollay 3.13. All chordal graphs are in $\mathcal{N}^{\prime}$.

Given a label-reading pair $(\mathscr{H}, \lambda)$ inducing $\phi: \pi_{1}(S) \rightarrow A(\Gamma)$, we will define the complexity of $(\mathscr{H}, \lambda)$. A label-reading pair $(\mathscr{H}, \lambda)$ is called normalized if the complexity of $(\mathscr{H}, \lambda)$ is minimal in the lexicographical ordering, among all the labelreading pairs inducing the same map up to conjugation in $A(\Gamma)$. Certain properties of a label-reading map can be more easily detected by looking at this normalized label-reading pairs. An edge $\{a, b\}$ of a graph $\Gamma$ is called bisimplicial if any vertex adjacent to $a$ is either equal or adjacent to any vertex that is adjacent to $b$ [13]. For $e \in E(\Gamma), \stackrel{\circ}{e}$ denote the interior of $e$.

Theorem 5.1. Let e be a bisimplicial edge of $\Gamma$. If $\Gamma \backslash \stackrel{\ominus}{e} \in \mathcal{N}^{\prime}$, then $\Gamma \in \mathcal{N}^{\prime}$.

A chordal bipartite graph is a graph that does not contain a triangle or an induced cycle of length at least 5. By definition, a chordal bipartite graph is not necessarily chordal. Any chordal bipartite graph can be obtained by successively attaching bisimplicial edges, starting from a discrete graph [13]. Using this, we prove:

Corollay 5.2. All chordal bipartite graphs are in $\mathcal{N}^{\prime}$.

In particular, if $\Gamma$ is chordal or chordal bipartite, then $A(\Gamma)$ does not contain a closed hyperbolic surface group. This first appeared in [21] and also follows from [5].

We will also prove that $\mathcal{N}^{\prime}$ is closed under a certain graph operation, called cocontraction [22]. Using this, a lower bound for $\mathcal{N}^{\prime}$ will be given. We will provide new examples of right-angled Artin groups that contain closed hyperbolic surface groups, by using co-contraction and results in [5]. Lastly, we will describe an equivalent formulation of Conjecture 1.1. A vertex of a graph $\Gamma$ is called simplicial, if the link of the vertex induces a complete subgraph of $\Gamma$.

Proposition 6.4. The following are equivalent.

(i) $\mathcal{N}$ is closed under complete graph amalgamation.

(ii) For any graph $\Gamma$, if the graph obtained by removing a simplicial vertex from $\Gamma$ is in $\mathcal{N}$, then $\Gamma$ is also in $\mathcal{N}$.

(iii) $\mathcal{N}^{\prime}=\mathcal{N}$.

Note. All the results in this article, except for Example 6.3, originally appeared in the Ph.D. thesis of the author [21]. After submission of his thesis, the author came 
to know that Crisp, Sageev and Sapir proved similar results to Corollary 3.13 and Theorem 5.1, where $\mathcal{N}^{\prime}$ is replaced by the presumably larger class $\mathcal{N}$ [5]. A special case of Lemma 3.10 can also be deduced from [5]. That is the case when there exists a fixed complete subgraph $K \leq \Gamma$ and a relative embedding $\phi: \pi_{1}(S) \rightarrow A(\Gamma)$ for some compact hyperbolic surface $S$, such that the image of each peripheral element of $\pi_{1}(S)$ is conjugate into $A(K)$. Their work is independent from the author, and the arguments are completely different.

Acknowledgement. I would like to thank my Ph.D. thesis advisor, Professor Andrew Casson for sharing his deep insights and valuable advice that guided me through this work. I am also grateful to Professor Alan Reid for many helpful comments on earlier versions of this article. Lastly, I am thankful for exceptionally kind and detailed feedback from an anonymous referee, particularly for hinting Remark 3.11.

\section{Label-reading maps}

In this section, we review basic properties of label-reading maps from surface groups into right-angled Artin groups. We owe most of the definitions and the results in this section to [6].

Recall our convention that we only consider oriented surfaces. Let $S$ be a compact surface with an arbitrarily chosen base in its interior. From the orientation of $S$, the boundary components of $S$ inherit canonical orientations so that $\sum\left[\partial_{i} S\right]=0$ in $H_{1}(S)$. We will often abbreviate a closed curve and a properly embedded arc on $S$ as a curve and an arc, respectively. We assume that a curve or an arc is given with an orientation, which is arbitrarily chosen unless specified. Suppose that $\Gamma$ is a graph. In $A(\Gamma)$, a letter means $v^{ \pm 1}$ for some $v \in V(\Gamma)$, and a word means a sequence of letters. A word represents an element in $A(\Gamma)$. Let $(\mathscr{H}, \lambda)$ be a label-reading pair on $S$ with the underlying graph $\Gamma$. Here, curves and arcs in $\mathscr{H}$ with the same label are allowed to intersect. This difference from [6] leaves most of the results and the arguments in [6] still valid. For $a \in V(\Gamma)$, an $a$-curve and an $a$-arc in $\mathscr{H}$ will mean a curve and an arc, respectively, labeled by $a$. For each based loop $\gamma$ transversely intersecting $\mathscr{H}$, one follows $\gamma$ starting from the base point; whenever $\gamma$ intersects $\alpha \in \mathscr{H}$, one records $\lambda(\gamma)$ or $\lambda(\gamma)^{-1}$ according to whether the orientation of $\gamma$ coincides with the transverse orientation of $\alpha$ or not. The word $w_{\gamma}$ thus obtained is called the label-reading of $\gamma$ with respect to $(\mathscr{H}, \lambda)$. Note that the label-reading $w_{\gamma}$ can also be defined if $\gamma$ is an oriented arc, instead of an oriented curve. The map $\phi: \pi_{1}(S) \rightarrow A(\Gamma)$, defined by $\phi([\gamma])=w_{\gamma}$, is called the label-reading map with respect to $(\mathscr{H}, \lambda)$.

Conversely, suppose that $\phi: \pi_{1}(S) \rightarrow A(\Gamma)$ is an arbitrary map. Write

$$
\pi_{1}(S)=\left\langle x_{1}, x_{2}, \ldots, x_{g}, y_{1}, y_{2}, \ldots, y_{g}, d_{1}, d_{2}, \ldots, d_{m} \mid \prod_{i=1}^{g}\left[x_{i}, y_{i}\right] \prod_{i=1}^{m} d_{i}\right\rangle .
$$

Here $d_{1}, d_{2}, \ldots, d_{m}$ correspond to the boundary components $\partial_{1} S, \partial_{2} S, \ldots, \partial_{m} S$ 
of $S$. Draw a dual van Kampen diagram $\Delta$ for the following word in $A(\Gamma)$ [26], [22]:

$$
w=\prod_{i=1}^{g}\left[\phi\left(x_{i}\right), \phi\left(y_{i}\right)\right] \prod_{i=1}^{m} \phi\left(d_{i}\right) .
$$

Recall this means that $\Delta$ is a disk along with a set of transversely oriented, properly embedded arcs labeled by $V(\Gamma)$, such that the label-reading of $\partial \Delta$ with respect to these arcs is $w$ (Figure 1 (a)). $\partial \Delta$ is subdivided into segments, so that the label-reading of each segment is a letter in $V(\Gamma)^{ \pm 1}$. Glue the boundary of $\Delta$ by identifying $\phi\left(x_{i}\right)$ with $\phi\left(x_{i}\right)^{-1}$, and also $\phi\left(y_{i}\right)$ with $\phi\left(y_{i}\right)^{-1}$, as in Figure $1(\mathrm{~b})$. Then one obtains $S$ back, with a set $\mathscr{H}$ of transversely oriented curves and $\operatorname{arcs}$ on $S$ and a labeling map $\lambda: \mathscr{H} \rightarrow V(\Gamma)$. It follows that $(\mathcal{H}, \lambda)$ is a label-reading pair, and $\phi$ is the label-reading map with respect to $(\mathscr{H}, \lambda)$ up to conjugation in $A(\Gamma)$. Moreover, if $\phi$

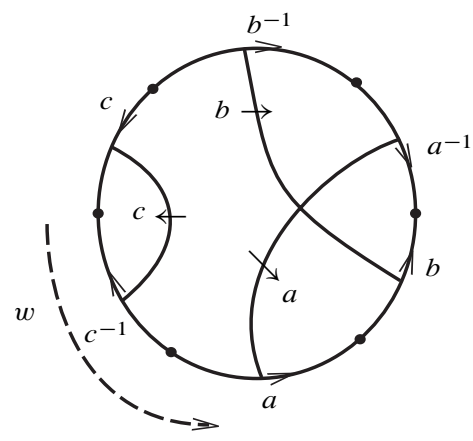

(a)

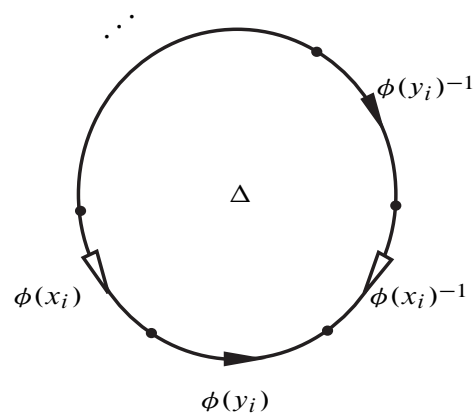

(b)

Figure 1. (a) A dual van Kampen diagram $\Delta$ for the word $w=c^{-1} a b a^{-1} b^{-1} c$ in the right-angled Artin group $\langle a, b, c \mid[a, b]=1\rangle$. (b) Identifying intervals on $\partial \Delta$.

is a relative embedding, then for each $i$ there exists a complete subgraph $K \leq \Gamma$ such that $\phi\left(d_{i}\right)=w_{i}^{\prime-1} w_{i} w_{i}^{\prime}$ for some $w_{i} \in A(K)$ and $w_{i}^{\prime} \in A(\Gamma)$. In this case, $(\mathcal{H}, \lambda)$ can be chosen so that any arc in $\mathscr{H}$ intersecting with a boundary component $\partial_{i} S$ is labeled by a letter in $V(K)$. This is achieved by gluing the words $w_{i}^{\prime}$ and $w_{i}^{\prime-1}$ in our construction. We summarize this as follows.

Proposition 2.1 ([6], [21]). Let $S$ be a compact surface and $\Gamma$ be a graph. Suppose that $\phi: \pi_{1}(S) \rightarrow A(\Gamma)$ is a map.

(1) $\phi$ is a label-reading map with respect to some label-reading pair $(\mathcal{H}, \lambda)$ with the underlying graph $\Gamma$.

(2) If $\phi$ is a relative embedding, then one can find $(\mathcal{H}, \lambda)$ in (1) satisfying the following: for each boundary component $\partial_{i} S$, there exists a complete subgraph 
$K \leq \Gamma$ such that any arc in $\mathscr{H}$ intersecting with $\partial_{i} S$ is labeled by a vertex in $V(K)$.

From now on, whenever we are given with a relative embedding $\phi: \pi_{1}(S) \rightarrow$ $A(\Gamma)$ with respect to a label-reading pair $(\mathcal{H}, \lambda)$, we will implicitly assume that $(\mathscr{H}, \lambda)$ satisfies the property described in Proposition $2.1(2)$.

Notation 2.2. Let $S$ be a compact surface.

(1) If $\alpha$ and $\beta$ are closed curves, $\alpha \sim \beta$ means $\alpha$ and $\beta$ are freely homotopic. If $A$ and $B$ are subsurfaces of $S, A \sim B$ means $A$ and $B$ are isotopic in $S$.

(2) If $\alpha$ and $\beta$ are properly embedded arcs, $\alpha \sim \beta$ means $\alpha$ and $\beta$ are homotopic, by a homotopy leaving the endpoints of $\alpha$ and $\beta$ on the boundary of $S$ (but not requiring the endpoints to be fixed).

(3) Let $A \subseteq S$. We write $\alpha \leadsto A$ and say $\alpha$ is homotopic into $A$, if $\alpha \sim \beta$ for some $\beta \subseteq A$; in particular, if $A=\partial S$ we say that $\alpha$ is homotopic into the boundary of $S$.

(4) $i(\alpha, \beta)=\min \left\{\alpha^{\prime} \cap \beta^{\prime} \mid \alpha \sim \alpha^{\prime}\right.$ and $\left.\beta \sim \beta^{\prime}\right\}$.

We say two maps $\phi, \psi: \pi_{1}(S) \rightarrow A(\Gamma)$ are equivalent if $\phi=i \circ \psi$ for some inner-automorphism $i: A(\Gamma) \rightarrow A(\Gamma)$. Note that for a fixed label-reading pair, a base change does not alter the equivalence class of the corresponding label-reading map $\pi_{1}(S) \rightarrow A(\Gamma)$. We also say two label-reading pairs are equivalent if they induce equivalent label-reading maps. There are certain simplifications on $(\mathcal{H}, \lambda)$ that do not change the equivalence class (see [6] for details and proofs).

Lemma 2.3 ([6]). Let $(\mathscr{H}, \lambda)$ and $\left(\mathscr{H}^{\prime}, \lambda^{\prime}\right)$ be label-reading pairs on $S$ with the underlying graph $\Gamma$. The label-reading maps induced by $(\mathcal{H}, \lambda)$ and $\left(\mathcal{H}^{\prime}, \lambda^{\prime}\right)$ are equivalent, if any of the following is satisfied.

(1) $\mathscr{H}^{\prime}$ is obtained by removing null-homotopic curves in $\mathscr{H}$.

(2) $\mathscr{H}^{\prime}$ is obtained by removing $\alpha \in \mathscr{H}$, for some $\alpha \leadsto \partial S$.

(3) Suppose that $\alpha, \beta \in \mathscr{H}$ intersect at $p$ and have the same label a. Alter $\alpha$ and $\beta$ on a neighborhood $D$ of $p$ so that we get $\alpha^{\prime}$ and $\beta^{\prime}$ which are labeled by $a$ and do not intersect in $D$. Transverse orientations of $\alpha^{\prime}$ and $\beta^{\prime}$ are determined by those of $\alpha$ and $\beta .\left(\mathscr{H}^{\prime}, \lambda^{\prime}\right)$ is the label-reading pair thus obtained (Figure 2(a)).

(4) Suppose that $\alpha, \beta \in \mathscr{H}$ bound a bigon. Alter $\alpha$ and $\beta$ on a neighborhood of the bigon so that we get $\alpha^{\prime}$ and $\beta^{\prime}$ which do not intersect in that neighborhood. The labels of $\alpha^{\prime}$ and $\beta^{\prime}$ are equal to those of $\alpha$ and $\beta$, respectively. $\left(\mathcal{H}^{\prime}, \lambda^{\prime}\right)$ is the label-reading pair thus obtained (Figure $2(\mathrm{~b})$ ).

Remark 2.4. Let $(\mathscr{H}, \lambda)$ be a label-reading pair on a compact surface $S$, inducing a label-reading map $\phi: \pi_{1}(S) \rightarrow A(\Gamma)$. 


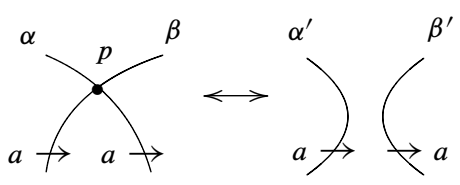

(a)

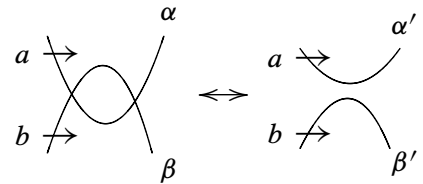

(b)

Figure 2. Homotopies that do not change the equivalence class of a label-reading pair. Note that (b) is allowed only when $a=\lambda(\alpha)$ and $b=\lambda(\beta)$ are equal or adjacent in $\Gamma$.

(1) By Lemma 2.3, we will always assume that curves and arcs in $\mathscr{H}$ are neither null-homotopic nor homotopic into the boundary. Moreover, curves and arcs in $\mathscr{H}$ will be assumed to be minimally intersecting [2]. Curves and arcs of the same label are assumed to be disjoint, unless stated otherwise.

(2) Let $\gamma$ be a curve or an arc on $S$, such that the endpoints (meaning the base point if $\gamma$ is a loop) are not on $\mathscr{H}$. If $\gamma$ is not transverse to $\mathscr{H}$, define the label-reading $w_{\gamma}$ by $w_{\gamma}=w_{\gamma^{\prime}}$ for some $\gamma^{\prime} \sim \gamma$ such that $\gamma^{\prime}$ has the same endpoints as $\gamma$, and $\gamma^{\prime}$ is transverse to $\mathscr{H}$. This definition of $w_{\gamma} \in A(\Gamma)$ does not depend on the choice of $\gamma^{\prime}$.

\section{3. $\mathcal{N}^{\prime}$ is closed under complete graph amalgamation}

Recall that $\mathcal{N}^{\prime}$ denotes the class of graphs, the right-angled Artin groups on which do not allow relative embeddings of compact hyperbolic surface groups (Definition 1.2). Let $\Gamma$ be a graph and $S$ be a compact hyperbolic surface. Recall that $x \in \pi_{1}(S)$ is called peripheral if $x=[\alpha]$ for some $\alpha$ homotopic into $\partial S$. We note that an embedding $\phi: \pi_{1}(S) \rightarrow A(\Gamma)$ is a relative embedding if, for each peripheral $x \in$ $\pi_{1}(S)$, there exists a complete subgraph $K \leq \Gamma$ such that $\phi(x)$ is conjugate into $A(K)$. In this section, we examine basic combinatorial properties of $\mathcal{N}^{\prime}$, and prove that $\mathcal{N}^{\prime}$ is closed under complete graph amalgamation. Roughly speaking, a key idea for the proof is that commutativity is scarce in hyperbolic surface groups. The following is immediate from the fact that any two elements in a compact hyperbolic surface group generate $\mathbb{Z}$ or a free group of rank 2 .

Lemma 3.1. Let $S$ be a compact hyperbolic surface, and $x$ and $y$ be commuting elements of $\pi_{1}(S)$. Then there exists $c \in \pi_{1}(S)$ such that $x, y \in\langle c\rangle$. If $x$ and $y$ are further assumed to be represented by essential simple closed curves, then either $x=y$ or $x=y^{-1}$.

Let $\Gamma_{1}$ and $\Gamma_{2}$ be graphs. The disjoint union of $\Gamma_{1}$ and $\Gamma_{2}$ is denoted by $\Gamma_{1} \sqcup \Gamma_{2}$. 
We define $\operatorname{JOIN}\left(\Gamma_{1}, \Gamma_{2}\right)$ to be the graph obtained by taking the disjoint union of $\Gamma_{1}$ and $\Gamma_{2}$ and adding the edges in $\left\{\left\{v_{1}, v_{2}\right\} \mid v_{1} \in V\left(\Gamma_{1}\right), v_{2} \in V\left(\Gamma_{2}\right)\right\}$. This means,

$$
\operatorname{JOIN}\left(\Gamma_{1}, \Gamma_{2}\right)=\overline{\overline{\Gamma_{1}} \sqcup \overline{\Gamma_{2}}} .
$$

Proposition 3.2. If $\Gamma_{1}, \Gamma_{2} \in \mathcal{N}^{\prime}$, then $\operatorname{JoIN}\left(\Gamma_{1}, \Gamma_{2}\right) \in \mathcal{N}^{\prime}$.

Proof. Suppose that $\Gamma=\operatorname{JOIN}\left(\Gamma_{1}, \Gamma_{2}\right) \notin \mathcal{N}^{\prime}$. One can find a relative embedding $\phi: \pi_{1}(S) \rightarrow A(\Gamma) \cong A\left(\Gamma_{1}\right) \times A\left(\Gamma_{2}\right)$ for some hyperbolic surface $S$. Let $(\mathcal{H}, \lambda)$ be a label-reading pair inducing $\phi$, and $p_{i}: A(\Gamma) \rightarrow A\left(\Gamma_{i}\right)$ be the projection map.

We claim that $p_{1} \circ \phi$ or $p_{2} \circ \phi$ is injective. Suppose not, and choose $1 \neq a_{1} \in$ $\operatorname{ker}\left(p_{1} \circ \phi\right)$ and $1 \neq a_{2} \in \operatorname{ker}\left(p_{2} \circ \phi\right)$. Write $\phi\left(a_{1}\right)=\left(1, b_{2}\right)$ and $\phi\left(a_{2}\right)=$ $\left(b_{1}, 1\right)$ for some non-trivial $b_{i} \in A\left(\Gamma_{i}\right), i=1,2 . \phi\left[a_{1}, a_{2}\right]=\left[\phi\left(a_{1}\right), \phi\left(a_{2}\right)\right]=$ $\left[\left(1, b_{2}\right),\left(b_{1}, 1\right)\right]=1$. Since $S$ is hyperbolic and $\phi$ is an embedding, $a_{1}, a_{2} \in\langle c\rangle$ for some $c \in \pi_{1}(S)$ (Lemma 3.1). Hence, $\left\langle\phi\left(a_{1}\right), \phi\left(a_{2}\right)\right\rangle \subseteq\langle\phi(c)\rangle \cong \mathbb{Z}$, which contradicts to $\mathbb{Z} \times \mathbb{Z} \cong\left\langle\left(1, b_{2}\right),\left(b_{1}, 1\right)\right\rangle=\left\langle\phi\left(a_{1}\right), \phi\left(a_{2}\right)\right\rangle$

Without loss of generality, we may assume that $p_{1} \circ \phi$ is injective. The labelreading map $\pi_{1}(S) \rightarrow A\left(\Gamma_{1}\right)$ obtained by removing curves and arcs in $\mathscr{H}$ labeled by $V\left(\Gamma_{2}\right)$ is injective. So $\Gamma_{1} \notin \mathcal{N}^{\prime}$.

Since $K_{1} \in \mathcal{N}^{\prime}$, it follows from Proposition 3.2 that $K_{n} \in \mathcal{N}^{\prime}$ for any $n$.

For the rest of this section, we will prove that $\mathcal{N}^{\prime}$ is closed under complete graph amalgamation. For a graph $\Gamma$, the set of all vertices adjacent to $a \in V(\Gamma)$ will be denoted by $\operatorname{LINK}(a)$.

Definition 3.3 ([14]). A vertex $a$ of a graph $\Gamma$ is called simplicial if LINK $(a)$ induces a complete subgraph of $\Gamma$.

A set of pairwise non-adjacent vertices in a graph is said to be independent.

Lemma 3.4. Let $\Gamma$ and $\Gamma^{\prime}$ be graphs such that $\Gamma^{\prime}$ is obtained by removing a set of independent simplicial vertices in $\Gamma$. If $\Gamma^{\prime} \in \mathcal{N}^{\prime}$, then $\Gamma \in \mathcal{N}^{\prime}$.

Proof. Let $\Gamma^{\prime}$ be the induced subgraph of $\Gamma$ on $V(\Gamma) \backslash\left\{a_{1}, \ldots, a_{r}\right\}$, where $a_{1}, \ldots, a_{r}$ are independent simplicial vertices of $\Gamma$. Suppose that $\Gamma \notin \mathcal{N}^{\prime}$.

First, consider the case when $r=1$. Let $a=a_{1}$. One can find a compact hyperbolic surface $S$ and a relative embedding $\phi: \pi_{1}(S) \rightarrow A(\Gamma)$ induced by a label-reading pair $(\mathscr{H}, \lambda)$. Put $\mathscr{H}_{a}=\lambda^{-1}(a)$.

Case 1. $\mathscr{H}_{a}$ consists of simple closed curves only.

Choose a connected component $S^{\prime}$ of $S \backslash\left(\cup \mathscr{H}_{a}\right)$, so that $S^{\prime}$ is hyperbolic. The curves and arcs in the set $(\cup \mathcal{H}) \cap S^{\prime}$ naturally inherit transverse orientations and labels from those of $(\mathscr{H}, \lambda)$, and so determine a label-reading pair $\left(\mathscr{H}^{\prime}, \lambda^{\prime}\right)$ inducing $\phi^{\prime}: \pi_{1}\left(S^{\prime}\right) \rightarrow A\left(\Gamma^{\prime}\right) . \phi^{\prime}$ is injective, since $\phi^{\prime}$ is a restriction of $\phi$ up to equivalence. 
A simple closed curve in $\mathscr{H}_{a}$ intersects with a curve in $\mathscr{H}$ labeled by a vertex in $\operatorname{LINK}(a)$. Each boundary component $\partial_{i} S^{\prime}$ of $S^{\prime}$ either is a boundary component of $S$, or comes from a curve in $\mathscr{H}_{a}$. In the latter case, any curve in $\mathscr{H}^{\prime}$ intersecting with $\partial_{i} S^{\prime}$ must be labeled by a vertex in $\operatorname{LINK}(a)$. Since LINK $(a)$ induces a complete graph in $\Gamma^{\prime}, \phi^{\prime}$ is a relative embedding. This implies $\Gamma^{\prime} \notin \mathcal{N}^{\prime}$.

Case 2. $\mathscr{H}_{a}$ contains a properly embedded arc $\alpha$.

Suppose that $\alpha$ joins the boundary components $\partial_{1} S$ and $\partial_{2} S$. Here, $\alpha$ is an essential arc, but it is possible that $\partial_{1} S=\partial_{2} S$. Since $\phi$ is a relative embedding, any curve or arc in $\mathscr{H}$ intersecting with $\partial_{1} S$ or $\partial_{2} S$ is labeled by a vertex in $\operatorname{LINK}(a) \cup\{a\}$. From the definition of a label-reading pair, the label-reading of $\alpha$ is in $\langle\operatorname{LINK}(a)\rangle$. Choose $\delta_{1} \sim \partial_{1} S$ and $\delta_{2} \sim \partial_{2} S$, such that $\delta_{1}$ and $\delta_{2}$ have the same basepoint, and transversely intersect $\mathscr{H}$. Moreover, we assume that $\delta_{1}$ and $\delta_{2}$ are sufficiently close to $\partial_{1} S$ and $\alpha \cdot \partial_{2} S \cdot \alpha^{-1}$ respectively, so that $w_{\delta_{1}}$ and $w_{\delta_{2}}$ are in $\langle\operatorname{LINK}(a) \cup\{a\}\rangle$ (Figure 3). Since $a$ is simplicial, $\langle\operatorname{LINK}(a) \cup\{a\}\rangle$ is free abelian, and $\phi\left(\left[\left[\delta_{1}\right],\left[\delta_{2}\right]\right]\right)=$ $\left[w_{\delta_{1}}, w_{\delta_{2}}\right]=1$. The injectivity of $\phi$ implies $\left[\left[\delta_{1}\right],\left[\delta_{2}\right]\right]=1$, which is impossible unless $\delta_{1} \sim \delta_{2}^{ \pm 1}$ and $S$ is an annulus (Lemma 3.1).

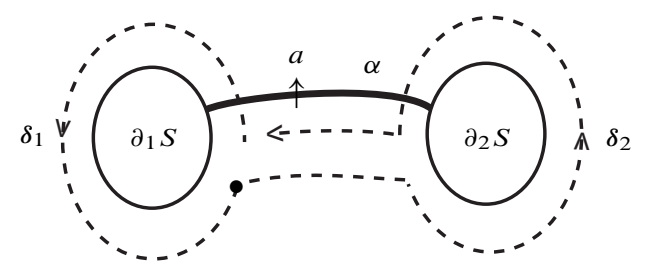

Figure 3

In the case when $r>1$, note that $a_{r}$ is a simplicial vertex of the induced subgraph on $V(\Gamma) \backslash\left\{a_{1}, a_{2}, \ldots, a_{r-1}\right\}$. An inductive argument shows that $\Gamma^{\prime} \notin \mathcal{N}^{\prime}$.

Using the next two lemmas, we will prove a general fact (Lemma 3.7) on the fundamental group of a hyperbolic surface with boundary. For a set $X$, the period of a finite sequence $f:\{1,2, \ldots, M\} \rightarrow X$ is the smallest positive number $p$ such that $f(i)=f(i+p)$ whenever $i$ and $i+p$ are in $\{1,2, \ldots, M\}$. The following combinatorial lemma asserts that if two finite sequences coincide at consecutive terms the number of which is as large as the sum of the periods, then one sequence is a translation of the other.

Lemma 3.5. Let $X$ be a set, and $M_{1}, M_{2}>0$. For $i=1,2$, let $A_{i}=\left\{1,2, \ldots, M_{i}\right\}$, and $f_{i}: A_{i} \rightarrow X$ be a finite sequence with the period $p_{i}$. Suppose that there exist integers $u$ and $v$ such that for each $i=v+1, v+2, \ldots, v+p_{1}+p_{2}$, we have $i \in A_{1}, u+i \in A_{2}$ and $f_{1}(i)=f_{2}(u+i)$. Then $p_{1}=p_{2}$, and $f_{1}(i)=f_{2}(u+i)$ whenever $i \in A_{1}, u+i \in A_{2}$.

Proof. We may assume that $p_{1} \leq p_{2}$. Suppose that $i, i+p_{1} \in A_{2}$. There exists some $q$ such that $u+v+1 \leq i+p_{2} q \leq u+v+p_{2}$. Then $f_{2}\left(i+p_{1}\right)=f_{2}\left(i+p_{1}+p_{2} q\right)=$ 
$f_{1}\left(i+p_{1}+p_{2} q-u\right)=f_{1}\left(i+p_{2} q-u\right)=f_{2}\left(i+p_{2} q\right)=f_{2}(i)$. Note that we used the conditions that $v+1+p_{1} \leq i+p_{1}+p_{2} q-u \leq v+p_{1}+p_{2}$ and $v+1 \leq$ $i+p_{2} q-u \leq v+p_{2}$. This shows that $p_{1}$ is also the period of $f_{2}$, and so, $p_{1}=p_{2}$. Now suppose that $i \in A_{1}$ and $u+i \in A_{2}$. For some $q^{\prime}, v+1 \leq i+p_{2} q^{\prime} \leq v+p_{2}$. Hence, $f_{1}(i)=f_{1}\left(i+p_{2} q^{\prime}\right)=f_{2}\left(u+i+p_{2} q^{\prime}\right)=f_{2}(u+i)$.

Recall that two elements in a free group are said to be independent if they do not have non-trivial conjugate powers. In the following lemma, the special case when $u_{1}=u_{2}=\cdots=u_{m}$ was first proved in [1], and further generalized to any word-hyperbolic group in [16]. The following proof uses a similar idea to [16].

Lemma 3.6. Let $F$ be a free group. Suppose $u_{1}, \ldots, u_{m} \in F \backslash\{1\}$, satisfying that any pair $u_{i}$ and $u_{j}$ are either equal or independent. Set $u_{0}=u_{m}$. Choose $b_{1}, \ldots, b_{m} \in F$ such that $u_{i-1}=u_{i}$ only if $\left[b_{i}, u_{i}\right] \neq 1$. Then there exists $N>0$ such that for any $\left|n_{1}\right|, \ldots,\left|n_{m}\right|>N, b_{1} u_{1}^{n_{1}} b_{2} u_{2}^{n_{2}} \ldots b_{m} u_{m}^{n_{m}}$ is non-trivial in $F$.

Proof. We may assume that each $u_{i}$ is cyclically reduced and not a proper power. For $g \in F,|g|$ denotes the word-length of $g$. Let $N$ be a sufficiently large integer which will be determined later in the proof, and $\left|n_{1}\right|, \ldots,\left|n_{m}\right|>N$. Suppose that $w=b_{1} u_{1}^{n_{1}} b_{2} u_{2}^{n_{2}} \ldots b_{m} u_{m}^{n_{m}}$ is trivial in $F$. Consider a dual van Kampen diagram $\Delta$ of $w$. The boundary $\partial \Delta$ is divided into segments, each of which intersects with only one properly embedded arc. For each $i$, the interval $u_{i}^{n_{i}}$ on $\partial \Delta$ intersects with $\left|n_{i}\right|\left|u_{i}\right|$ arcs. Since $u_{i}$ is cyclically reduced, no arc intersects $u_{i}^{n_{i}}$ twice. Hence, there exists $j$ such that there are at least

$$
M_{i}=\frac{1}{m}\left(\left|n_{i}\right|\left|u_{i}\right|-\sum_{k}\left|b_{k}\right|\right)
$$

$\operatorname{arcs}$ joining $u_{i}^{n_{i}}$ and $u_{j}^{n_{j}}$. If two arcs $\alpha$ and $\beta$ join $u_{i}^{n_{i}}$ and $u_{j}^{n_{j}}$, then so does any arc between $\alpha$ and $\beta$. This means $u_{i}^{n_{i}}$ and $u_{j}^{-n_{j}}$ have a common subword of length at least $M_{i}$. The word $u_{i}^{n_{i}}$ is a finite sequence of the period $\left|u_{i}\right|$. Since $\left|u_{i}\right| \neq 0$, one can choose a sufficiently large $N$ such that $M_{i}>\sum_{k}\left|u_{k}\right|$. Lemma 3.5 implies that $u_{i}$ is a cyclic conjugation of $u_{j}^{ \pm 1}$. By the independence of $u_{i}$ and $u_{j}, u_{i}=u_{j}$. Note that such $j$ exists for any $i$. So, if one chooses such a pair $(i, j)$ which is innermost, then $j=i+1$ or $j=i-1$. Assume that $j=i-1$. In $\Delta$, some arcs join an interval of the form $u_{i}^{k}$ in $u_{i}^{n_{i}}$ to an interval in $u_{i-1}^{n_{i-1}}$. By cutting $\Delta$ along these arcs, one obtains another dual van Kampen diagram for some word of the form $u_{i-1}^{p} b_{i} u_{i}^{q}=u_{i}^{p} b_{i} u_{i}^{q}$. Here, $u_{i-1}^{p}$ and $u_{i}^{q}$ are subwords of of $u_{i-1}^{n_{i-1}}$ and $u_{i}^{n_{i}}$, respectively. We have $u_{i}^{p} b_{i} u_{i}^{q}=1$, which is a contradiction to $\left[b_{i}, u_{i}\right] \neq 1$.

For a compact surface $S, D(S)$ denotes the double of $S$ along $\partial S$. The following lemma is well known when the surface $S$ has only one boundary component [28], [1]. 
Lemma 3.7. Let $S$ be a surface with the boundary components $\partial_{1} S, \ldots, \partial_{m} S$. $q: D(S) \rightarrow S$ denotes the natural quotient map. Let $T_{i}: D(S) \rightarrow D(S)$ be the Dehn twist along $\partial_{i} S \subseteq D(S)$. Then for any $x \in \pi_{1}(D(S)) \backslash\{1\}$ there exists $N>0$ such that whenever $\left|n_{1}\right|, \ldots,\left|n_{m}\right|>N,\left(q \circ T_{1}^{n_{1}} \circ \cdots \circ T_{m}^{n_{m}}\right)_{*}(x) \neq\{1\}$.

Proof. Put $\pi_{1}(S)=\left\langle x_{1}, \ldots, x_{g}, y_{1}, \ldots, y_{g}, d_{1}, \ldots, d_{m} \mid \prod\left[x_{i}, y_{i}\right] \prod d_{i}=1\right\rangle$, where $d_{i}$ is represented by a loop freely homotopic to $\partial_{i} S$. Let $h: S^{\prime} \rightarrow S$ be a homeomorphism, such that $D(S)=S \cup S^{\prime}$ glued along $\partial_{i} S=\partial_{i} S^{\prime}$ for each $i$. Let $v$ be the base point of $S$, and $v^{\prime}$ be its image in $S^{\prime}$. One can find arcs $\delta_{1}, \delta_{2}, \ldots, \delta_{m}$ joining $v$ to $v^{\prime}$ in $D(S)$ such that (Figure 4),

(i) $\delta_{i}$ and $\partial_{j} S$ intersect if and only if $i=j$,

(ii) $\left[q \circ T_{1}^{n_{1}} \circ \cdots \circ T_{m}^{n_{m}}\left(\delta_{i}\right)\right]=\left[q \circ T_{i}^{n_{i}}\left(\delta_{i}\right)\right]=d_{i}^{n_{i}}$.

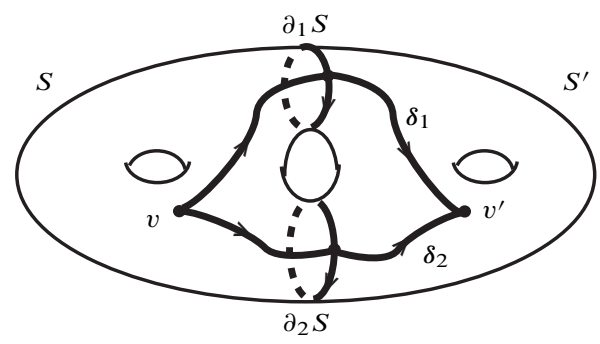

Figure 4. The double of a surface.

Choose any $x \in \pi_{1}(D(S)) \backslash\{1\}$. If $x$ is in $\pi_{1}(S) \leq \pi_{1}(D(S))$, then $(q \circ$ $\left.T_{1}^{n_{1}} \circ \cdots \circ T_{m}^{n_{m}}\right)_{*}(x)=x \neq 1$. Hence, we may assume $x \notin \pi_{1}(S)$. For some $l \geq 1$ and $1 \leq i_{1}, i_{2}, \ldots, i_{2 l} \leq m, x$ can be represented as a concatenation of arcs $x=\beta_{1} \cdot \delta_{i_{1}} \cdot \beta_{2} \cdot \delta_{i_{2}}^{-1} \ldots \beta_{2 l} \cdot \delta_{i_{2 l}}^{-1}$ possibly after a conjugation. Here, $\beta_{1}, \beta_{3}, \beta_{5}, \ldots$ are loops in $S$ based at $v$, and $\beta_{2}, \beta_{4}, \beta_{6}, \ldots$ are loops in $S^{\prime}$ based at $v^{\prime}$. By choosing the minimal $l$, one may assume that if $i_{k-1}=i_{k}$ then $\beta_{k}$ is not homotopic into $\partial_{i_{k}} S=\partial_{i_{k}} S^{\prime}$. One can write

$$
\left(q \circ T_{1}^{n_{1}} \circ \cdots \circ T_{m}^{n_{m}}\right)_{*}(x)=\left[\beta_{1}\right] d_{i_{1}}^{n_{i_{1}}}\left[h\left(\beta_{2}\right)\right] d_{i_{2}}^{-n_{i_{2}}} \ldots\left[h\left(\beta_{2 l}\right)\right] d_{i_{2 l}}^{-n_{i_{2 l} l}} .
$$

By applying Lemma 3.6 to the free group $\pi_{1}(S)$, one sees that $\left(q \circ T_{1}^{n_{1}} \circ \ldots \circ\right.$ $\left.T_{m}^{n_{m}}\right)_{*}(x) \neq 1$ if $n_{1}, \ldots, n_{m}$ are all sufficiently large.

From now on, we denote the set of maximal complete subgraphs of $\Gamma$ by $\mathcal{K}(\Gamma)$. We define a graph operation, called simplicial extension.

Definition 3.8. Let $\Gamma$ be a graph. Define the simplicial extension of $\Gamma$, denoted by $\Gamma^{*}$, to be the graph having the following vertex and edge sets:

(i) $V\left(\Gamma^{*}\right)=V(\Gamma) \sqcup\left\{v_{K, u} \mid K \in \mathcal{K}(\Gamma), u \in V(K)\right\}$;

(ii) $E\left(\Gamma^{*}\right)=E(\Gamma) \sqcup\left\{\left\{v_{K, u}, u^{\prime}\right\} \mid K \in \mathcal{K}(\Gamma), u, u^{\prime} \in V(K)\right\}$. 
$\Gamma^{*}$ is obtained from $\Gamma$ by adding a simplicial vertex (denoted by $v_{K, u}$ ) for each pair of a maximal complete subgraph $K$ and a vertex $u$ of $K$; see Figure 5. We first make a graph theoretical observation regarding simplicial extensions.

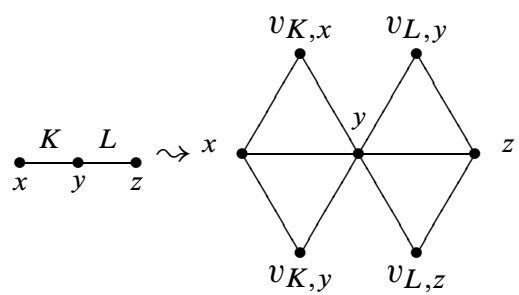

(a) $P_{3}$ and $P_{3}^{*}$

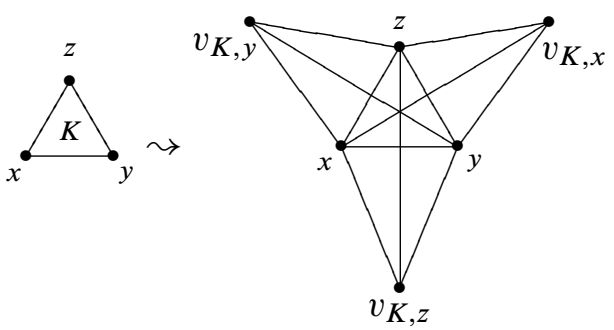

(b) $K_{3}$ and $K_{3}^{*}$

Figure 5. Examples of simplicial extensions.

Lemma 3.9 (Decomposing $\Gamma^{*}$ ). Suppose that $\Gamma$ is a complete graph amalgamation of $\Gamma_{1}$ and $\Gamma_{2}$. Then $\Gamma^{*}$ is a complete graph amalgamation of $\Gamma_{1}^{\prime}$ and $\Gamma_{2}^{\prime}$ for some $\Gamma_{1}^{\prime}, \Gamma_{2}^{\prime}$ such that

(i) $\Gamma_{i} \leq \Gamma_{i}^{\prime} \leq \Gamma_{i}^{*}$,

(ii) $V\left(\Gamma_{i}^{\prime}\right) \backslash V\left(\Gamma_{i}\right)$ is a set of independent simplicial vertices of $\Gamma_{i}^{\prime}$.

Proof. Let $K=\Gamma_{1} \cap \Gamma_{2}$. We may assume that $K \neq \varnothing$. Note that $\mathcal{K}\left(\Gamma_{1}\right) \cap \mathcal{K}\left(\Gamma_{2}\right) \subseteq$ $\{K\}$ and $\mathcal{K}(\Gamma) \subseteq \mathcal{K}\left(\Gamma_{1}\right) \cup \mathcal{K}\left(\Gamma_{2}\right) \subseteq \mathcal{K}(\Gamma) \cup\{K\}$.

Case 1. $K \in \mathcal{K}\left(\Gamma_{1}\right) \cup \mathcal{K}\left(\Gamma_{2}\right)$.

We may assume $K \in \mathcal{K}\left(\Gamma_{1}\right)$. Then, $K$ is maximal in $\Gamma_{2}$ if and only if $K$ is maximal in $\Gamma$. Combining this with $\mathcal{K}\left(\Gamma_{2}\right) \subseteq \mathcal{K}\left(\Gamma_{1}\right) \cup \mathcal{K}\left(\Gamma_{2}\right) \subseteq \mathcal{K}(\Gamma) \cup\{K\}$, one has $\mathcal{K}\left(\Gamma_{2}\right) \subseteq \mathcal{K}(\Gamma)$. Define $\Gamma_{1}^{\prime}$ to be the graph obtained from $\Gamma_{1}^{*}$ by removing the simplicial vertices $\left\{v_{K, u} \mid u \in V(K)\right\}$. Put $\Gamma_{2}^{\prime}=\Gamma_{2}^{*} . \Gamma_{1}^{\prime} \cap \Gamma_{2}^{\prime}=\Gamma_{1} \cap \Gamma_{2}=K$. Moreover, $V\left(\Gamma^{*}\right) \subseteq V\left(\Gamma_{1}^{\prime} \cup \Gamma_{2}^{\prime}\right) \cup\left\{v_{K, u} \mid u \in V(K)\right\}$. Since $\mathcal{K}\left(\Gamma_{1}\right) \backslash\{K\} \subseteq \mathcal{K}(\Gamma)$ and $\mathcal{K}\left(\Gamma_{2}\right) \subseteq \mathcal{K}(\Gamma), \Gamma_{1}^{\prime} \cup \Gamma_{2}^{\prime} \subseteq \Gamma^{*}$. If $K \notin \mathcal{K}(\Gamma)$, then $v_{K, u} \notin V\left(\Gamma^{*}\right)$ for each $u \in V(K)$, and so, $V\left(\Gamma^{*}\right) \subseteq V\left(\Gamma_{1}^{\prime} \cup \Gamma_{2}^{\prime}\right)$. If $K \in \mathcal{K}(\Gamma)$, then $K \in \mathcal{K}\left(\Gamma_{2}\right)$ and $v_{K, u} \in V\left(\Gamma_{2}^{\prime}\right)$ for each $u \in V(K)$; this implies $V\left(\Gamma^{*}\right) \subseteq V\left(\Gamma_{1}^{\prime} \cup \Gamma_{2}^{\prime}\right) \cup\left\{v_{K, u} \mid u \in\right.$ $V(K)\}=V\left(\Gamma_{1}^{\prime} \cup \Gamma_{2}^{\prime}\right)$. It follows that $\Gamma^{*}=\Gamma_{1}^{\prime} \cup \Gamma_{2}^{\prime}$.

Case 2. $K \notin \mathcal{K}\left(\Gamma_{1}\right) \cup \mathcal{K}\left(\Gamma_{2}\right)$.

In this case, $\mathcal{K}(\Gamma)=\mathcal{K}\left(\Gamma_{1}\right) \sqcup \mathcal{K}\left(\Gamma_{2}\right)$. Hence, $\Gamma^{*}=\Gamma_{1}^{*} \cup \Gamma_{2}^{*}$ and $\Gamma_{1}^{*} \cap \Gamma_{2}^{*}=$ $\Gamma_{1} \cap \Gamma_{2}=K$. Set $\Gamma_{1}^{\prime}=\Gamma_{1}^{*}$ and $\Gamma_{2}^{\prime}=\Gamma_{2}^{*}$.

Lemma 3.10 is a key step for the proof of Theorem 3.12. The lemma states that a relative embedding of a compact hyperbolic surface group into $A(\Gamma)$ can be "promoted" to an embedding of a closed hyperbolic surface group into $A\left(\Gamma^{*}\right)$. 
Lemma 3.10. Let $\Gamma$ be a graph. Then $\Gamma \in \mathcal{N}^{\prime}$ if and only if $\Gamma^{*} \in \mathcal{N}$.

Proof. $\Longrightarrow$ : Suppose that $\Gamma \in \mathcal{N}^{\prime} . \Gamma$ is obtained from $\Gamma^{*}$ by removing a set of independent simplicial vertices. By Lemma 3.4, $\Gamma^{*} \in \mathcal{N}^{\prime} \subseteq \mathcal{N}$.

$\Longleftarrow$ : Suppose that $\Gamma \notin \mathcal{N}^{\prime}$. Fix a compact hyperbolic surface $S$ and a relative embedding $\phi: \pi_{1}(S) \rightarrow A(\Gamma)$, which is a label-reading map with respect to $(\mathcal{H}, \lambda)$. Denote the boundary components of $S$ by $\partial_{1} S, \partial_{2} S, \ldots, \partial_{m} S$. Recall $\partial S$ is oriented so that $\sum\left[\partial_{i} S\right]=0$ in $H_{1}(S)$. Since $\phi$ is a relative embedding, we may assume that for each boundary component $\partial_{i} S$ of $S$, there exists a complete subgraph $K$ of $\Gamma$ such that the curves and the arcs in $\mathscr{H}$ intersecting with $\partial_{i} S$ are labeled by $V(K)$.

Let $S^{\prime}$ be a surface homeomorphic to $S$ by a homeomorphism $g: S \rightarrow S^{\prime}$. Put $\partial_{i} S^{\prime}=g\left(\partial_{i} S\right)$. We consider $D(S)$ as the union of $S, S^{\prime}$ and the annuli $A_{1}, A_{2} \ldots, A_{m}$. Here, $A_{i}$ is parametrized by $f_{i}:[-1,1] \times S^{1} \rightarrow A_{i}$, such that $f_{i}\left(-1 \times S^{1}\right)$ and $f_{i}\left(1 \times S^{1}\right)$ are glued to $\partial_{i} S$ and $\partial_{i} S^{\prime}$, respectively. We will define a label-reading pair $\left(\mathscr{H}^{\prime}, \lambda^{\prime}\right)$ on $D(S)$, which restricts to $(\mathscr{H}, \lambda)$ on $S$. To do this, we will write $\mathscr{H}^{\prime}=\mathscr{H}_{1}^{\prime} \cup \mathscr{H}_{2}^{\prime} \cup \mathscr{H}_{3}^{\prime}$ as follows.

$\mathscr{H}_{1}^{\prime}$ will be the collection of the simple closed curves $\gamma$ and $g(\gamma)$, for all simple closed curves $\gamma \in \mathscr{H}$. Here, we let $\gamma$ and $g(\gamma)$ in $\mathscr{H}_{1}^{\prime}$ inherit the label and the transverse orientation of $\gamma \in \mathscr{H}$.

To define $\mathscr{H}_{2}^{\prime}$, let $\gamma \in \mathscr{H}$ be a properly embedded arc, joining $\partial_{i} S$ and $\partial_{j} S$. Let $f_{i}\left(-1 \times p_{i}\right)$ and $f_{j}\left(-1 \times p_{j}\right)$ be the intersection of $\gamma$ with $\partial_{i} S$ and $\partial_{j} S$, respectively. There exists a simple closed curve $\tilde{\gamma}$ on $D(S)$ obtained by taking a concatenation of $\gamma, f_{j}\left([-1,1] \times p_{j}\right), g\left(\gamma^{-1}\right)$ and $f_{i}\left([-1,1] \times p_{i}\right)$. Again, we let $\tilde{\gamma}$ inherit the label and the transverse orientation of $\gamma$, and define $\mathscr{H}_{2}^{\prime}$ to be the collection of all such simple closed curves $\tilde{\gamma}$ on $D(S)$, where $\gamma$ ranges over all properly embedded $\operatorname{arcs}$ in $\mathscr{H}$.

Now we define $\mathscr{H}_{3}^{\prime}$ as follows. Consider any boundary component $\partial_{i} S$, and let $\beta_{1}, \beta_{2}, \ldots, \beta_{s} \subseteq S$ be the properly embedded arcs in $\mathscr{H}$ intersecting with $\partial_{i} S$. There exists a (possibly non-unique) maximal complete subgraph $K \leq \Gamma$ such that $\lambda\left(\beta_{j}\right) \in V(K)$ for all $j$. We choose disjoint essential simple closed curves $\alpha_{1}, \ldots, \alpha_{s}$ in the interior of $A_{i}$, and let $\lambda^{\prime}\left(\alpha_{j}\right)=v_{K, \lambda\left(\beta_{j}\right)} \in V\left(\Gamma^{*}\right)$, for each $j$. Moreover, we let the transverse orientation of $\alpha_{j}$ be from $f_{i}\left(-1 \times S^{1}\right)$ to $f_{i}\left(1 \times S^{1}\right)$, if the transverse orientation of $\beta_{j}$ coincides with the orientation of $\partial_{i} S$, and be the opposite otherwise (Figure 6(a)). Let $\mathscr{H}_{3}^{\prime}$ be the collection of all such $\alpha_{j}$ 's, for all the boundary components $\partial_{1} S, \ldots, \partial_{m} S$. In this way, we have defined a set of transversely oriented curves and arcs $\mathscr{H}^{\prime}=\mathscr{H}_{1}^{\prime} \cup \mathscr{H}_{2}^{\prime} \cup \mathscr{H}_{3}^{\prime}$ and a labeling $\lambda^{\prime}: \mathscr{H}^{\prime} \rightarrow V\left(\Gamma^{*}\right)$.

Let $\phi^{\prime}: \pi_{1}(D(S)) \rightarrow A\left(\Gamma^{*}\right)$ be the label-reading map with respect to $\left(\mathscr{H}^{\prime}, \lambda^{\prime}\right)$. Fix any $n>0$. Define $p_{n}: A\left(\Gamma^{*}\right) \rightarrow A(\Gamma)$ by $p_{n}(a)=a$ for $a \in V(\Gamma)$, and $p_{n}\left(v_{K, u}\right)=u^{n}$ for $K \in \mathcal{K}(\Gamma)$ and $u \in V(K)$. Let $T_{i}$ be the Dehn twist of $D(S)$ along $\partial_{i} S$, and $T=T_{1} \circ T_{2} \circ \cdots \circ T_{m}$. 
Claim. The following diagram commutes up to equivalence.

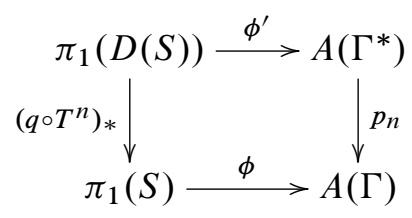

Note that $\phi \circ q_{*}$ is the label-reading map with respect to the pair $\left(\mathscr{H}_{1}^{\prime} \cup \mathscr{H}_{2}^{\prime}, \lambda^{\prime}\right)$. Similarly, $\phi \circ\left(q \circ T^{n}\right)_{*}$ is the label-reading map with respect to the pair consisting of the set $T^{-n}\left(\mathscr{H}_{1}^{\prime} \cup \mathscr{H}_{2}^{\prime}\right)$, and the labeling map $\lambda^{\prime} \circ T^{n}$.

Let $\alpha \in \mathscr{H}_{3}^{\prime}$ be any simple closed curve inside an annulus, say $A_{i}$. Write $\lambda^{\prime}(\alpha)=$ $v_{K, u}$ for some $K \in \mathcal{K}(\Gamma)$ and $u \in V(K)$. Consider $n$ copies of disjoint essential simple closed curves $\tilde{\alpha}_{1}, \tilde{\alpha}_{2}, \ldots, \tilde{\alpha}_{n} \subseteq A_{i}$, with the same transverse orientation as $\alpha$. Label $\tilde{\alpha}_{1}, \tilde{\alpha}_{2}, \ldots, \tilde{\alpha}_{n}$ by $u$. Define $\mathcal{C}_{n}$ to be the collection of all such $\tilde{\alpha}_{j}$ 's, for all the simple closed curves $\alpha \in \mathscr{H}_{3}^{\prime}$. Then $\mathscr{H}_{1}^{\prime} \cup \mathscr{H}_{2}^{\prime} \cup \mathcal{C}_{n}$ with the transverse orientations and the labeling defined so far determines a label-reading map $\psi: \pi_{1}(D(S)) \rightarrow A(\Gamma)$. Note that two curves or arcs of the same label are not necessarily disjoint in this label-reading pair (Figure 6(b)). From the construction, $\psi=p_{n} \circ \phi^{\prime}$.

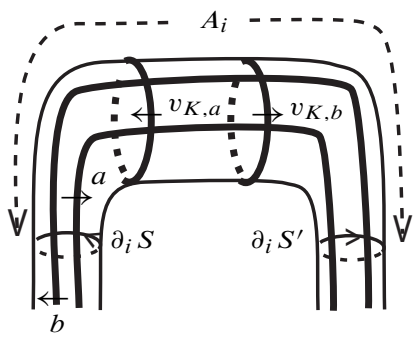

(a) $\mathscr{H}^{\prime}=\mathscr{H}_{1}^{\prime} \cup \mathscr{H}_{2}^{\prime} \cup \mathscr{H}_{3}^{\prime}$

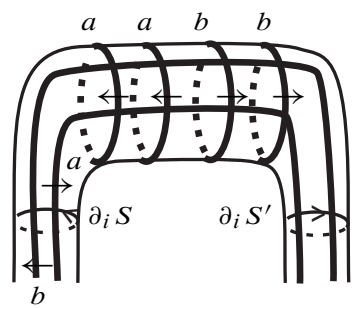

(b) $\mathscr{H}_{1}^{\prime} \cup \mathscr{H}_{2}^{\prime} \cup \mathscr{C}_{n}$

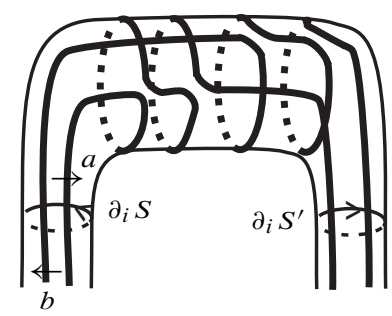

(c) $T^{-n}\left(\mathscr{H}_{1}^{\prime} \cup \mathscr{H}_{2}^{\prime}\right)$

Figure 6. Defining a label-reading pair $\left(\mathscr{H}^{\prime}, \lambda^{\prime}\right)$ on $D(S)$. (b) and (c) show equivalent labelreading pairs. 
From Lemma 2.3 (3), one immediately sees that $\psi$ is equivalent to the labelreading map with respect to $\left(T^{-n}\left(\mathscr{H}_{1}^{\prime} \cup \mathscr{H}_{2}^{\prime}\right), \lambda^{\prime} \circ T^{n}\right)$ (Figure $\left.6(\mathrm{c})\right)$. Therefore, $p_{n} \circ \phi^{\prime}(x)$ is equivalent to $\phi \circ\left(q \circ T^{n}\right)_{*}(x)$. The claim is proved.

Now suppose that $x$ is a non-trivial element in $\pi_{1}(D(S))$. By Lemma 3.7, there exists $n \geq 0$ such that $\left(q \circ T^{n}\right)_{*}(x)=\left(q \circ T_{1}^{n} \circ T_{2}^{n} \circ \cdots T_{m}^{n}\right)_{*}(x) \neq 1$. The injectivity of $\phi$ and the commutativity of the diagram above imply that $\phi^{\prime}(x)$ is non-trivial. Hence, $\phi^{\prime}$ is injective, and so, $\Gamma^{*} \notin \mathcal{N}$.

Remark 3.11. (1) One can also see that $\phi^{\prime}$ is injective in the above proof by a method from [5], rather than resorting to Lemma 3.7. The labels of the curves inside two different annuli $A_{i}$ and $A_{j}$ are either simplicial vertices joined to the same maximal complete subgraph of $\Gamma$, or disjoint and independent. Now suppose that $\phi^{\prime}[\alpha]=1$ for some $[\alpha] \in \pi_{1}(D(S)) \backslash\{1\}$. By the solution to the word problem for right-angled Artin groups, $\alpha$ has an essential subarc $\beta$ (in $S$ or $S^{\prime}$ ) satisfying the following: $\beta$ intersects boundary components $\partial_{i} S$ and $\partial_{j} S$, such that the curves inside $A_{i}$ and $A_{j}$ are labeled by simplicial vertices joined to the same maximal complete subgraph $K$ of $\Gamma$ and the label-reading by $(\mathscr{H}, \lambda)$ of $\beta$ is in $A(K)$ (note that $i=j$ may occur). This implies that any arc intersecting with $\partial_{i} S$ or $\partial_{j} S$ is labeled by a vertex of $K$. Orient $\beta$ from $\partial_{i} S$ to $\partial_{j} S$. Then the label-reading by $(\mathcal{H}, \lambda)$ of $\beta \cdot \partial_{j} S \cdot \beta^{-1}$ and $\partial_{i} S$ are both in $A(K)$, and so, commute. This will contradict to the injectivity of $\phi$.

(2) Lemma 3.6 is interesting in its own right. Let $\Gamma$ be a graph with two vertices $v_{1}$ and $v_{2}$, and multi-edges $e_{1}, e_{2}, \ldots, e_{r}$ joining $v_{1}$ and $v_{2}$. Fix independent words $u_{1}, \ldots, u_{r}$ in a non-abelian free group $F$. Define $G$ to be a graph of groups on $\Gamma$ by choosing two copies of $F$ as the vertex groups and letting the edge group $G_{i} \cong \mathbb{Z}$ be glued along the copies of $\left\langle u_{i}\right\rangle$ for each $i$. Then Lemma 3.6 shows that $G$ is fully residually free.

Theorem 3.12. $\mathcal{N}^{\prime}$ is closed under complete graph amalgamation.

Proof. Let $\Gamma, \Gamma_{1}$ and $\Gamma_{2}$ be graphs, such that $\Gamma$ is a complete graph amalgamation of $\Gamma_{1}$ and $\Gamma_{2}$. We will show that $\Gamma \in \mathcal{N}^{\prime}$ if and only if $\Gamma_{1}, \Gamma_{2} \in \mathcal{N}^{\prime}$.

$\Longrightarrow$ : Obvious, since $\Gamma_{i} \leq \Gamma$.

$\Longleftarrow$ : Assume that $\Gamma \notin \mathcal{N}^{\prime}$. By Lemma 3.10, $\Gamma^{*} \notin \mathcal{N} . \Gamma^{*}$ is a complete graph amalgamation of induced subgraphs $\Gamma_{1}^{\prime} \geq \Gamma_{1}$ and $\Gamma_{2}^{\prime} \geq \Gamma_{2}$, as in Lemma 3.9. By Lemma 1.3, we may assume $\Gamma_{1}^{\prime} \notin \mathcal{N}^{\prime}$. Since $\Gamma_{1}^{\prime}$ can be obtained by adding independent simplicial vertices to $\Gamma_{1}$, Lemma 3.4 implies $\Gamma_{1} \notin \mathcal{N}^{\prime}$

Corollary 3.13. Any chordal graph is in $\mathcal{N}^{\prime}$.

Proof. Recall that each complete graph is in $\mathcal{N}^{\prime}$ (Proposition 3.2). For each chordal graph $\Gamma$, either $\Gamma$ is complete or $\Gamma$ can be written as a complete graph amalgamation $\Gamma=\Gamma_{1} \cup \Gamma_{2}$ of proper induced subgraphs $\Gamma_{1}$ and $\Gamma_{2}$ [8]. By Theorem 3.12, an inductive argument shows that $\Gamma$ is in $\mathcal{N}^{\prime}$. 
In particular, $A(\Gamma)$ does not contain a closed hyperbolic surface group if $\Gamma$ is chordal. The condition that the underlying graph $\Gamma$ is chordal is equivalent to two important group theoretic properties on $A(\Gamma)$. Namely, $\Gamma$ is chordal, if and only if $A(\Gamma)$ is coherent [9], if and only if $A(\Gamma)$ has a free commutator subgroup [27].

\section{Normalized label-reading pairs}

In this section, we let $\Gamma$ be a graph and $S$ be a compact surface. For a given labelreading pair on $S$ with the underlying graph $\Gamma$, we will consider a simplification (called, normalization) of the label-reading pair, without changing the equivalence class of the induced label-reading map (Definition 4.2). Lemma 4.3 and 4.7 will be crucially used in Section 5.

Definition 4.1 (Regular label-reading pair). A label-reading pair $(\mathscr{H}, \lambda)$ on $S$ with the underlying graph $\Gamma$ is called regular, if the following are satisfied.

(i) The induced label-reading map $\phi: \pi_{1}(S) \rightarrow A(\Gamma)$ is injective.

(ii) The curves and the arcs in $\mathscr{H}$ are neither null-homotopic nor homotopic into the boundary.

(iii) Any curves and arcs in $\mathscr{H}$ are minimally intersecting. This means that for any $\alpha \neq \beta$ in $\mathscr{H},|\alpha \cap \beta|=i(\alpha, \beta)$.

(iv) Two curves or arcs of the same label do not intersect.

(v) For each boundary component $\partial_{i} S$, there exists a complete subgraph $K \leq \Gamma$ such that any arc $\alpha$ intersecting with $\partial_{i} S$ satisfies $\lambda(\alpha) \in V(K)$.

From Proposition 2.1 and Lemma 2.3, any relative embedding $\phi: \pi_{1}(S) \rightarrow A(\Gamma)$ is induced by a regular label-reading pair $(\mathscr{H}, \lambda)$ possibly after a conjugation in $A(\Gamma)$. This is the first step to simplify a given label-reading pair.

Definition 4.2 (Normalized label-reading pair). (1) Let $(\mathscr{H}, \lambda)$ be a regular labelreading pair on a hyperbolic surface $S$, and $\mathscr{B}$ be the set of properly embedded arcs in $\mathscr{H}$. Define the complexity of $\mathscr{H}$ to be the 4 -tuple of the nonnegative integers

$$
\begin{aligned}
& c(\mathcal{H}, \lambda) \\
& =\left(|(\cup \mathcal{B}) \cap \partial S|, \sum_{a \in V(\Gamma)}\left|\lambda^{-1}(a) / \sim\right|, \sum_{a \in V(\Gamma)}\left|\lambda^{-1}(a) \cap \mathscr{B} / \sim\right|, \sum_{\substack{\alpha, \beta \in \mathcal{H} \\
\alpha \neq \beta}}|\alpha \cap \beta|\right)
\end{aligned}
$$

where $\sim$ denotes the homotopy equivalence relation on $\mathscr{H}$, and also on $\mathscr{B}$. We denote the lexicographical ordering of the complexities by $\preceq$.

(2) A regular label-reading pair $(\mathcal{H}, \lambda)$ is normalized if for any other regular label-reading pair $\left(\mathscr{H}^{\prime}, \lambda^{\prime}\right)$ which is equivalent to $(\mathcal{H}, \lambda), c(\mathscr{H}, \lambda) \preceq c\left(\mathscr{H}^{\prime}, \lambda^{\prime}\right)$. 
In the above definition, $|(\cup \mathscr{B}) \cap \partial S|$ denotes the number of intersection points between $\partial S$ and the $\operatorname{arcs}$ in $\mathscr{B}$. This means, $|(\cup \mathscr{B}) \cap \partial S|=|(\cup \mathscr{H}) \cap \partial S|$ is the number of the endpoints of arcs in $\mathscr{B}$. It is obvious that any regular label-reading pair is equivalent to a normalized one. We start with a simple observation on normalized label-reading pairs.

Lemma 4.3 (Normalization I). Let $(\mathcal{H}, \lambda)$ be a normalized label-reading pair on a compact hyperbolic surface $S$ with the underlying graph $\Gamma$. $B$ denotes the set of properly embedded arcs in $\mathcal{H}$. If $\alpha, \beta \in \mathscr{B}$ have the same label and intersect with $\partial_{i} S$ for some $i$, then the transverse orientation of $\alpha$ and that of $\beta$ induce the same orientation on $\partial_{i} S$ at their intersections with $\partial_{i} S$. In particular, each properly embedded arc in $\mathscr{H}$ intersects with two distinct boundary components of $S$.

Proof. Let $a=\lambda(\alpha)=\lambda(\beta)$. Suppose that the transverse orientations of $\alpha$ and $\beta$ do not induce the same orientation on $\partial_{i} S$ at their intersection points $\left\{P_{\alpha}, P_{\beta}\right\}$. By choosing a nearest one among such pairs of intersection points on $\partial_{i} S$, we may assume a component of $\partial_{i} S \backslash\left\{P_{\alpha}, P_{\beta}\right\}$ does not intersect with any $a$-arc (Figure 7 (a)). By Lemma 2.3, one can reduce $|(\cup \mathcal{B}) \cap \partial S|$ by 2 without changing the equivalence class of $(\mathscr{H}, \lambda)$, if one replaces $\alpha$ and $\beta$ by another curve or arc $\alpha^{\prime}$ as in Figure 7 (b). Note that this new label-reading pair can be further simplified to become regular, again by Lemma 2.3.

Remark 4.4. During the proof of Lemma 4.3, one might have increased the number of homotopy classes of simple closed curves in $\lambda^{-1}(a)$, when $\alpha$ and $\beta$ are replaced by $\alpha^{\prime}$. But the proof is still valid, since we are considering the lexicographical ordering of the complexity.

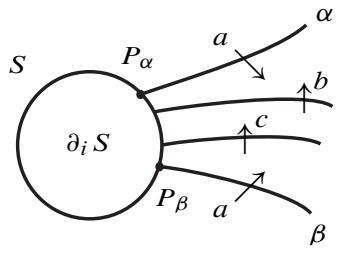

(a)

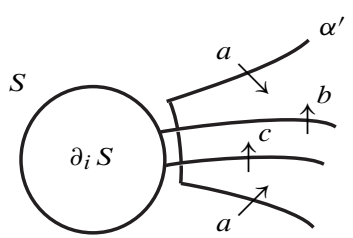

(b)

Figure 7. Reducing complexity. In (a), the labels $b$ and $c$ are adjacent to $a$ in $\Gamma$ by the regularity of $(\mathscr{H}, \lambda)$. Hence in (b), the intersections of $\alpha^{\prime}$ with $b$ - and $c$-arcs are allowed to occur. Consequently, $\alpha$ and $\beta$ can be replaced by $\alpha^{\prime}$ without changing the equivalence class of the label-reading pair.

To state properties of normalized label-reading pairs, it will be convenient to define certain terms regarding a set of disjoint properly embedded $\operatorname{arcs}$ on $S$, as follows. Let $I$ denote the unit interval $[0,1]$. 
Definition 4.5 (Strips and channels). Let $\mathcal{A}$ be a set of disjoint properly embedded arcs on a compact surface $S$.

(1) We choose an embedding $\eta_{\alpha}: I \times[-1,1] \rightarrow S$ for each $\operatorname{arc} \alpha \in \mathcal{A}$, such that the following conditions hold.

(i) $\eta_{\alpha}(I \times s)$ is a properly embedded arc for each $s \in[-1,1]$.

(ii) $\alpha \subseteq \eta_{\alpha}(I \times(-1,1))$.

(iii) If $\alpha \sim \alpha^{\prime} \in \mathcal{A}$, then $\eta_{\alpha}=\eta_{\alpha^{\prime}}$.

(iv) If $\alpha \nsim \alpha^{\prime} \in \mathcal{A}$, then the image of $\eta_{\alpha}$ and that of $\eta_{\alpha^{\prime}}$ are disjoint.

We call $\left\{\eta_{\alpha} \mid \alpha \in \mathcal{A}\right\}$ a set of strips for $\mathcal{A}$. For convenience, the image of $\eta_{\alpha}$ is also denoted by $\eta_{\alpha}$ when there is no danger of confusion.

(2) A channel is a connected component of $\left(\cup_{\alpha \in \mathcal{A}} \eta_{\alpha}\right) \cup \partial S$. For $\alpha \in \mathcal{A}$, we denote the unique channel containing $\alpha$ by $\operatorname{ch}(\alpha)$. An induced simple closed curve of $\alpha$ is a boundary component $\hat{\alpha}$ of the closure of $S \backslash \operatorname{ch}(\alpha)$ such that $\hat{\alpha} \cap \eta_{\alpha} \neq \varnothing$ and $\hat{\alpha} \nsubseteq \partial S$. Note that there exist at most two induced simple closed curves of $\alpha$ for each $\alpha \in \mathcal{A}$.

(3) An arc $\alpha \in \mathcal{A}$ is one-sided with respect to $\mathcal{A}$, if $\eta_{\alpha}(I \times\{-1\})$ and $\eta_{\alpha}(I \times\{1\})$ are contained in the same induced simple closed curve (see Figure 8 ).

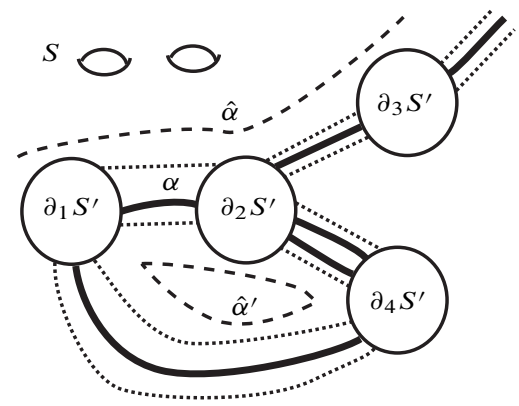

(a) $\alpha$ is not one-sided.

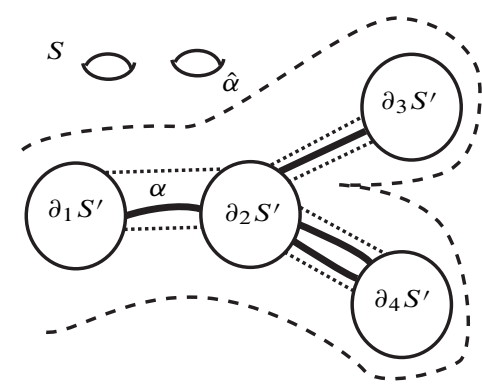

(b) $\alpha$ is one-sided.

Figure 8. Strips and channels of a set $\mathcal{A}$ of disjoint properly embedded arcs. Curves in $\mathcal{A}$ are drawn bold. The dotted arcs bound strips, along with some intervals on $\partial S$. $\hat{\alpha}$ and $\hat{\alpha}^{\prime}$ are induced simple closed curves. They do intersect $\partial S$ and the boundaries of strips, but for convenience of drawing, the figures show curves parallel to $\hat{\alpha}$ and $\hat{\alpha}^{\prime}$, in the interior of the surface. In (a), $\hat{\alpha}$ and $\hat{\alpha}^{\prime}$ are distinct induced simple closed curves of $\alpha \in \mathcal{A}$. In (b), $\hat{\alpha}$ is the unique induced simple closed curve of $\alpha$.

Remark 4.6. Let $\mathcal{A}$ be a set of disjoint properly embedded arcs on a compact surface $S$, and let $\alpha \in \mathcal{A}$.

(1) Any induced simple closed curve $\hat{\alpha}$ of $\alpha$ can be written as a concatenation of 
paths

$$
\hat{\alpha}=\alpha_{1}^{\prime} \cdot \delta_{1} \cdot \alpha_{2}^{\prime} \cdot \delta_{2} \cdot \alpha_{3}^{\prime} \ldots \delta_{r}
$$

such that

(i) $\alpha_{1}^{\prime} \sim \alpha$,

(ii) $\alpha_{i}^{\prime} \sim \alpha_{i}$ for some $\alpha_{i} \in \mathcal{A}$, and $\alpha_{i}^{\prime}$ is an interval on the boundary of the strip of $\alpha_{i}$,

(iii) $\delta_{i}$ is an interval on a boundary component of $S$ that intersects with $\alpha_{i}^{\prime}$ and $\alpha_{i+1}^{\prime}$.

In particular, an induced simple closed curve consists of subarcs which lie on $\partial S$ or the boundaries of strips. Moreover, if $\alpha$ is not one-sided, then the transverse orientation of $\alpha$ uniquely determines a transverse orientation of $\hat{\alpha}$ that respects the homotopy $\alpha_{1}^{\prime} \sim \alpha$.

(2) For a sufficiently small closed regular neighborhood $N$ of $\operatorname{ch}(\alpha)$, there exist disjoint annuli $A_{1}, A_{2}, \ldots, A_{r}$ in the closure of $S \backslash \operatorname{ch}(\alpha)$, such that $N=$ $\operatorname{ch}(\alpha) \cup A_{1} \cup A_{2} \cup \cdots \cup A_{r}$. The intersection of each $A_{i}$ with $\operatorname{ch}(\alpha)$ is an induced simple closed curve, and conversely, any induced simple closed curve intersecting with $\operatorname{ch}(\alpha)$ is a boundary component of some $A_{i}$.

(3) Let $(\mathscr{H}, \lambda)$ be a label-reading pair on $S$ with the underlying graph $\Gamma$. Consider any arc $\alpha \in \mathscr{H}$, and let $a=\lambda(\alpha)$. Denote the set of $a$-arcs by $\mathcal{A}_{a}$. We may assume that the arcs in $\mathcal{A}_{a}$ are disjoint (Lemma 2.3 (3)). Then the strip, the channel, and the induced simple closed curves of $\alpha$ are defined to be those of $\alpha$ with respect to the set $\mathcal{A}_{a}$. Furthermore, $\alpha$ is said to be one-sided if it is one-sided with respect to $\mathcal{A}_{a}$.

Lemma 4.7 (Normalization II). Let $(\mathscr{H}, \lambda)$ be a normalized label-reading pair on a compact hyperbolic surface $S$ with the underlying graph $\Gamma$. Then each arc $\alpha$ in $\mathscr{H}$ is one-sided (see Remark 4.6(3)).

Proof. Let $a=\lambda(\alpha)$. For abbreviation, we simply let $\mathcal{A}$ denote the set of $a$-arcs. Suppose that there exists an arc $\alpha \in \mathcal{A}$, which is not one-sided in $\mathcal{A}$. Let $\hat{\alpha}$ be one of the two induced simple closed curves of $\alpha$ with respect to $\mathcal{A}$. Write $\hat{\alpha}=$ $\alpha_{1}^{\prime} \cdot \delta_{1} \cdot \alpha_{2}^{\prime} \cdot \delta_{2} \ldots \alpha_{r}^{\prime} \cdot \delta_{r}$, where $\delta_{i} \subseteq \partial S$, and $\alpha_{i}^{\prime}$ is a properly embedded arc homotopic to an $a$-arc $\alpha_{i} \in \mathcal{A}$, as in Remark 4.6(1). Here, $\alpha_{1}^{\prime} \sim \alpha$. The transverse orientation of $\hat{\alpha}$ is given by that of $\alpha$.

First, consider the case when no other curve in $\mathcal{A}$ is homotopic to $\alpha$. Choose an embedding $g: S^{1} \times I \rightarrow \overline{S \backslash \operatorname{ch}(\alpha)}$ such that $g\left(S^{1} \times\{0\}\right)=\hat{\alpha}$, as in Remark 4.6(2). Put $\beta=g\left(S^{1} \times\left\{\frac{1}{2}\right\}\right)$ and $\gamma=g\left(S^{1} \times\{1\}\right)$. One may assume that $\gamma$ is sufficiently close to $\hat{\alpha}$, so that if any $\gamma^{\prime} \in \mathcal{H}$ intersects with $\gamma$, then $\gamma^{\prime}$ also intersects with $\hat{\alpha}$. Let $\gamma$ have the transverse orientation which the homotopy $\gamma \sim \hat{\alpha}$ respects, and give $\beta$ the opposite orientation (Figure 9(a)). Label $\beta$ and $\gamma$ by $a$ and add them to $\mathscr{H}$; this results in a new label-reading pair $\left(\mathscr{H}_{1}, \lambda_{1}\right)$. Note that $\left(\mathscr{H}_{1}, \lambda_{1}\right)$ is equivalent to 
$(\mathcal{H}, \lambda)$, since the homotopic curves $\beta$ and $\gamma$ have the same label and the opposite transverse orientations.

Note that the transverse orientations of $\alpha_{2}, \alpha_{3}, \alpha_{4}, \alpha_{5}, \ldots$ are completely determined by that of $\alpha$, according to Lemma 4.3. Obtain another label-reading pair $\left(\mathscr{H}_{2}, \lambda_{2}\right)$ from $\left(\mathscr{H}_{1}, \lambda_{1}\right)$ by removing $\beta, \alpha, \alpha_{3}, \alpha_{5}, \ldots$ and adding $a$-arcs homotopic to $\alpha_{2}, \alpha_{4}, \ldots$. Here, newly added arcs will have the transverse orientations respecting the homotopies to $\alpha_{2}, \alpha_{4}, \ldots$, as in Figure 9 (c). One sees that $\left(\mathscr{H}_{2}, \lambda_{2}\right)$ is equivalent to $\left(\mathscr{H}_{1}, \lambda_{1}\right)$ by successive applications of Lemma 2.3 (3). Figure 9 (b) illustrates an intermediate step between $\left(\mathscr{H}_{1}, \lambda_{1}\right)$ and $\left(\mathscr{H}_{2}, \lambda_{2}\right)$.

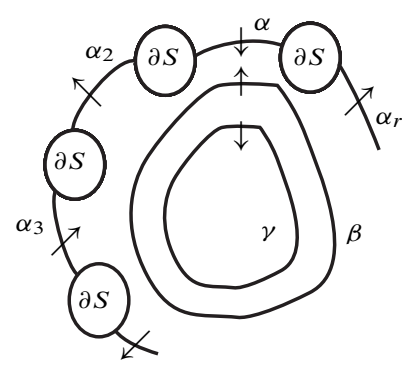

(a) $\left(\mathscr{H}_{1}, \lambda_{1}\right)$

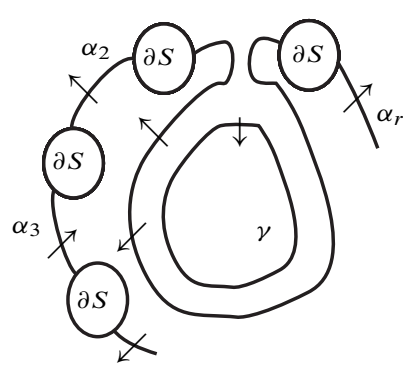

(b) a reduction of $\left(\mathscr{H}_{1}, \lambda_{1}\right)$

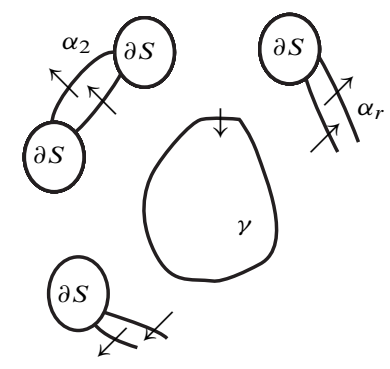

(c) $\left(\mathscr{H}_{2}, \lambda_{2}\right)$

Figure 9. All the curves and arcs drawn here are labeled by $a$.

Since $\alpha$ is not one-sided, $\alpha_{i} \not \alpha$ for each $i>1$. We have assumed that $\alpha$ is the only arc in its homotopy class, contained in $\mathcal{A}_{\text {. Hence, }}\left(\mathscr{H}_{2}, \lambda_{2}\right)$ does not contain any $a$-arc homotopic to $\alpha$. This means, $\mathscr{H}_{2}$ has a strictly smaller number of homotopy classes of properly embedded arcs, than $\mathscr{H}$ does. $\mathscr{H}_{2}$ has the same number of, or one more, homotopy classes of simple closed curves than $\mathscr{H}$ does, according to whether there exists any $a$-curve homotopic to $\gamma$ in $\mathscr{H}$ or not. This implies, $\left|\lambda_{2}^{-1}(a) / \sim\right| \leq\left|\lambda^{-1}(a) / \sim\right|$. Moreover, $|\mathcal{H} \cap \partial S|=\left|\mathscr{H}_{2} \cap \partial S\right|$. Hence, 
$c\left(\mathscr{H}_{2}, \lambda_{2}\right) \prec c(\mathscr{H}, \lambda)$. This is a contradiction to the minimality of $c(\mathcal{H}, \lambda)$.

In the case when there exist $l>1$ properly embedded arcs in $\mathscr{H}$ homotopic to $\alpha$, fix a small annulus $A \subseteq \overline{S \backslash \operatorname{ch}(\alpha)}$, of which $\hat{\alpha}$ is a boundary component. Consider a set of disjoint, transversely oriented, simple closed curves $\beta_{1}, \beta_{2}, \ldots, \beta_{l}, \gamma_{1}, \gamma_{2}, \ldots, \gamma_{l}$ contained in $A$ with this order, such that $\beta_{1}$ is the closest to $\hat{\alpha}$. Here, we let $\beta_{1}, \beta_{2}, \ldots, \beta_{l}$ have the opposite transverse orientations to that of $\hat{\alpha}$, and $\gamma_{1}, \gamma_{2}, \ldots, \gamma_{l}$ have the transverse orientations coinciding with that of $\hat{\alpha}$. By letting $\mathscr{H}_{1}=\mathscr{H} \cup$ $\left\{\beta_{1}, \beta_{2}, \ldots, \beta_{l}, \gamma_{1}, \gamma_{2}, \ldots, \gamma_{l}\right\}$, the same argument implies that $c(\mathcal{H}, \lambda)$ is not minimal.

Now we state a lemma regarding a set of disjoint properly embedded arcs on a compact surface, such that each arc is one-sided. In view of Lemma 4.7, this result will be applied to the study of normalized label-reading pairs in the next section. Note, the conclusion of Lemma 4.8 is not true without the hypothesis that each arc is one-sided.

Lemma 4.8. Let $\mathcal{A}$ be a set of disjoint properly embedded arcs on a surface $S$, such that each arc in $\mathcal{A}$ is one-sided. Denote the union of the boundary components of $S$ intersecting with arcs in $\mathcal{A}$ by $\partial^{*} S$. Fix $\alpha \in \mathcal{A}$, and let $\hat{\alpha}$ be the unique induced simple closed curve of $\alpha$. For a sufficiently small closed regular neighborhood $N$ of $\operatorname{ch}(\alpha)$, the following hold.

(1) $N$ has a unique boundary component, say $\hat{\alpha}^{\prime}$, that is not a boundary component of $S$. $\hat{\alpha}^{\prime}$ separates $S$, and $\hat{\alpha}^{\prime} \sim \hat{\alpha}$.

(2) Any properly embedded arc or closed curve, not intersecting with $(\cup \mathcal{A}) \cup \partial^{*} S$, can be homotoped into $S \backslash N$.

(3) If we further assume that $\hat{\alpha}$ is null-homotopic, then $\partial^{*} S=\partial S$ and any essential closed curve on $S$ intersects with $\cup \mathcal{A}$.

Proof. (1) We use the notations in Definition 4.5. We will say that a boundary component of $S$ or a strip of $\mathcal{A}$ is good if it intersects with $\hat{\alpha}$ (Remark 4.6(1)).

Claim 1. If a strip is good, then so is any boundary component of $S$ intersecting with that strip.

If a strip $\eta_{\beta}: I \times[-1,1] \rightarrow S$ is good for some $\beta \in \mathcal{A}$, then $\eta_{\beta}(I \times-1)$ or $\eta_{\beta}(I \times 1)$ is contained in $\hat{\alpha}$. Since $\beta$ is one-sided, $\eta_{\beta}(I \times\{-1,1\}) \subseteq \hat{\alpha}$. In particular, $\eta_{\beta}(\{0,1\} \times\{-1,1\}) \subseteq \hat{\alpha}$. Hence, the boundary components of $S$ that intersect with the good strip $\eta_{\beta}$ intersects with $\hat{\alpha}$.

Now we denote the boundary components of $S$ by $\partial_{1} S, \partial_{2} S, \ldots, \partial_{m} S$.

Claim 2. If $\partial_{i} S$ is good, then so is any strip intersecting with $\partial_{i} S$.

Suppose that $\partial_{i} S$ is good. $\hat{\alpha} \cap \partial_{i} S$ is a union of intervals on $\partial_{i} S$, and the endpoints of any of those intervals are contained in good strips. Assume that $\partial_{i} S$ also intersects 
with a strip that is not good. On $\partial_{i} S$, one can choose a nearest pair of a good strip $\eta_{\beta_{1}}$ and a strip $\eta_{\beta_{2}}$ that is not good, for some $\beta_{1}, \beta_{2} \in \mathcal{A}$. This implies, there exists a closed interval $u$ on $\partial_{i} S$ such that $u$ intersects with $\eta_{\beta_{1}}$ and $\eta_{\beta_{2}}$, but not with any other strips. Since $\eta_{\beta_{1}}$ is good, the unique induced simple closed curve of $\beta_{1}$ is $\hat{\alpha}$, and so, $u \subseteq \hat{\alpha}$. Since $u$ intersects with the induced simple closed curve of $\beta_{2}$, we have a contradiction to the assumption that $\eta_{\beta_{2}}$ is not good.

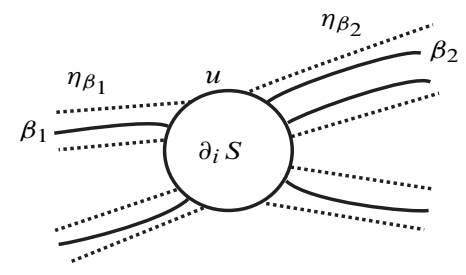

Figure 10. Proof of Claim 2.

By Claim 1 and 2, if $\partial_{i} S$ and $\partial_{j} S$ are connected by an arc in $\mathcal{A}$, and $\partial_{i} S$ is good, then so is $\partial_{j} S$. Since $\operatorname{ch}(\alpha)$ is connected, it follows that a boundary component of $S$ or a strip is good if and only if it is contained in $\operatorname{ch}(\alpha)$.

Now choose any component $\kappa$ of $\partial N \backslash \partial S$. There exists $\beta \in \mathcal{A}$, such that $\kappa$ and the induced simple closed curve $\hat{\beta}$ of $\beta$ bound an annulus contained in the closure of $N \backslash \operatorname{ch}(\alpha)$, and $\hat{\beta} \subseteq \operatorname{ch}(\alpha)$ (Remark 4.6 (2)). The strip $\eta_{\beta}$ of $\beta$ intersects with $\hat{\beta}$. Since $\hat{\beta} \subseteq \operatorname{ch}(\alpha), \eta_{\beta} \cap \operatorname{ch}(\alpha) \neq \varnothing$. This implies that $\eta_{\beta} \subseteq \operatorname{ch}(\alpha)$, and so, $\eta_{\beta}$ is good; that is, $\eta_{\beta}$ intersects also with $\hat{\alpha}$. Since $\beta$ is assumed to be one-sided, $\hat{\beta}=\hat{\alpha}$. Hence, $\kappa$ is the unique boundary component of $N$ that bounds an annulus with $\hat{\alpha}$. This proves that $\partial N$ contains only one component that is not in $\partial S$.

Note that $N$ is a proper subsurface of $S$ such that the frontier, namely $\kappa$, is connected. This implies $\kappa$ separates $S$.

(2) Suppose that $\gamma$ is a curve or an $\operatorname{arc}$ on $S$, not intersecting with $(\cup \mathcal{A}) \cup \partial^{*} S$. Let $\mathcal{A}_{0}$ be a minimal set of arcs in $\mathcal{A}$ satisfying the following:

for each $\beta \in \mathcal{A}$, there uniquely exists $\beta_{0} \in \mathcal{A}_{0}$ such that $\beta \sim \beta_{0}$.

Case 1. $\gamma$ is a closed curve.

We have

$$
\gamma \subseteq S \backslash(\cup \mathcal{A}) \subseteq S \backslash\left(\cup \mathcal{A}_{0}\right) \sim S \backslash\left(\cup_{\beta \in \mathcal{A}} \eta_{\beta}\right) \subseteq \overline{S \backslash \operatorname{ch}(\alpha)} \sim \overline{S \backslash N} .
$$

The first homotopy is obtained by enlarging each arc in $\mathcal{A}_{0}$ to a strip, and the second one is a deformation retract of the annuli discussed in Remark 4.6 (2) onto circles. Clearly, $\overline{S \backslash N}$ can be homotoped into $S \backslash N$.

Case 2. $\gamma$ is a properly embedded arc.

The argument for this case is almost the same as Case 1. One has only to show that that there exists a homotopy that sends $\gamma$ into $S \backslash N$, leaving the endpoints on $\partial S$. For this, we choose $N$ as a sufficiently small regular neighborhood of $\operatorname{ch}(\alpha)$ such 
that $\partial \gamma \cap N=\varnothing$. This is possible since $\gamma$ does not intersect $\partial^{*} S$. Then we have only to note that the homotopies in the proof of Case 1 do not move $\partial \gamma$.

(3) Suppose that $\hat{\alpha} \sim 0$. From (1), there exists $\kappa \sim \hat{\alpha}$ such that $\partial N \subseteq\{\kappa\} \cup \partial^{*} S$. Since $\kappa$ separates, one can write $S=N \cup S^{\prime}$ such that $N \cap S^{\prime}=\kappa . N$ contains at least one boundary component of $S$, namely any of the boundary components that $\alpha$ intersects. Hence, $N$ is not a disk. Now for $\kappa$ to be null-homotopic, $S^{\prime}$ must be a disk and $\partial S \subseteq \partial N$. So $\partial N=\{\kappa\} \cup \partial S$, and $\partial^{*} S=\partial S$.

Let $\gamma$ be any closed curve, not intersecting with any arc in $\mathcal{A}$. By (2), $\gamma$ is homotopic into $S \backslash N$, which is the interior of $S^{\prime}$. This implies that $\gamma$ is nullhomotopic.

\section{Adding bisimplicial edges}

An edge $\{a, b\}$ is bisimplicial if any vertex $\operatorname{in} \operatorname{LINK}(a)$ is either equal or adjacent to any vertex in $\operatorname{LINK}(b)$. For an edge $e$ of a graph, $\stackrel{\ominus}{e}$ denotes the interior of $e$. In this section, we prove the following theorem.

Theorem 5.1. Let e be a bisimplicial edge of a graph $\Gamma$. If $\Gamma \backslash \AA \in \mathcal{N}^{\prime}$, then $\Gamma \in \mathcal{N}^{\prime}$.

Proof. Write $e=\{a, b\}$, and let $\Gamma^{\prime}=\Gamma \backslash \stackrel{\ominus}{e}$. Assume that $\Gamma \notin \mathcal{N}^{\prime}$. One can find a compact hyperbolic surface $S$, and a relative embedding $\phi: \pi_{1}(S) \rightarrow A(\Gamma)$ with respect to a normalized label-reading pair $(\mathscr{H}, \lambda)$.

First, consider the case when $\lambda(\mathscr{H}) \subseteq \operatorname{LINK}(a) \cup\{a\} . \Gamma_{\text {LINK }}(a) \cup\{a\} \notin \mathcal{N}^{\prime}$, since the image of $\phi$ is in $A\left(\Gamma_{\mathrm{LINK}}(a) \cup\{a\}\right) . \Gamma_{\mathrm{LINK}}(a) \cup\{a\}$ is the join of the single vertex $a$ and $\Gamma_{\text {LINK }(a)}$. Since a single vertex is in $\mathcal{N}^{\prime}$, Proposition 3.2 implies that $\Gamma_{\text {LINK }(a)} \notin \mathcal{N}^{\prime}$. Note that $\Gamma_{\mathrm{LINK}}(a) \leq \Gamma_{V(\Gamma) \backslash\{a\}} \leq \Gamma^{\prime}$. Hence, $\Gamma^{\prime}$ is not in $\mathcal{N}^{\prime}$, which contradicts to the assumption. The case when $\lambda(\mathscr{H}) \subseteq \operatorname{LINK}(b) \cup\{b\}$ is similar.

Now assume that $\lambda(\mathscr{H}) \nsubseteq \operatorname{LINK}(a) \cup\{a\}$ and $\lambda(\mathscr{H}) \nsubseteq \operatorname{LINK}(b) \cup\{b\}$. We denote the boundary components of $S$ by $\partial_{1} S, \partial_{2} S, \ldots, \partial_{m} S$. For a based curve or arc $\gamma$ on $S$, we let $w_{\gamma}$ denote the label-reading of $\gamma$ with respect to the label-reading pair $(\mathcal{H}, \lambda)$ as in Section 2.

Claim 1. Suppose that $\alpha$ and $\beta$ are essential simple closed curves on $S$ such that $\alpha \cap \beta \neq \varnothing, w_{\alpha} \in\langle\operatorname{LINK}(a)\rangle$ and $w_{\beta} \in\langle\operatorname{LINK}(b)\rangle$. Then $\alpha \sim \beta^{ \pm 1}$.

We may choose the base point of $\pi_{1}(S)$ in $\alpha \cap \beta . \phi[[\alpha],[\beta]]=\left[w_{\alpha}, w_{\beta}\right]=1$, since any vertex in $\operatorname{LINK}(a)$ is equal or adjacent to any vertex in $\operatorname{LINK}(b)$. By Lemma 3.1, $\alpha \sim \beta^{ \pm 1}$. Here, we have assumed that $\alpha$ and $\beta$ are transverse to $\mathscr{H}$. If not, one may consider $\alpha^{\prime} \sim \alpha$ and $\beta^{\prime} \sim \beta$ such that $\alpha^{\prime}$ and $\beta^{\prime}$ are sufficiently close to $\alpha$ and $\beta$ respectively, and transversely intersecting $\mathscr{H}$. The claim is proved.

For $v \in V(\Gamma)$, recall that simple closed curves and properly embedded arcs in $\mathscr{H}$ labeled by $v$ are called $v$-curves and $v$-arcs, respectively. $\mathscr{C}_{v}$ and $\mathcal{A}_{v}$ will denote the 
set of $v$-curves and the set of $v$-arcs, respectively. Let $\partial^{v} S$ denote the union of the boundary components of $S$ that intersect with $v$-arcs.

Claim 2. $\left(\cup \mathcal{C}_{a}\right) \cap\left(\cup \mathcal{C}_{b}\right)=\varnothing$.

Suppose that $\alpha$ and $\beta$ intersect at a point $p$, for some $\alpha \in \mathscr{C}_{a}$ and $\beta \in \mathscr{C}_{b}$. One can find simple closed curves $\alpha_{1} \sim \alpha$ and $\beta_{1} \sim \beta$, intersecting at a point $p^{\prime}$ near $p$, such that $\alpha_{1}$ and $\beta_{1}$ are transverse to $\mathscr{H}$. By requiring that $\alpha_{1}$ is sufficiently close to $\alpha$, we may assume that the label-reading of $\alpha_{1}$ with the base point $p^{\prime}$, is same as the label-reading of $\alpha$ with a suitable choice of the base point. If $\gamma \in \mathscr{H}$ intersects with $\alpha$, then $\lambda(\gamma) \in \operatorname{LINK}(a)$, by the definition of a label-reading pair. Hence, $w_{\alpha_{1}} \in$ $\langle\operatorname{LINK}(a)\rangle$. Similarly, $w_{\beta_{1}} \in\langle\operatorname{LINK}(b)\rangle$. By Claim 1, $\alpha \sim \alpha_{1} \sim \beta_{1}^{ \pm 1} \sim \beta^{ \pm 1}$, which contradicts to the assumption that curves and arcs in $\mathscr{H}$ are minimally intersecting (Remark 2.4(1)).

Claim 3. If $\alpha \in \mathcal{A}_{a}$ and $\beta \in \mathcal{A}_{b}$, then $\alpha \nsim \beta$.

Suppose that an $a$-arc $\alpha$ and a $b$-arc $\beta$ are homotopic. They join the same pair of boundary components, say $\partial_{1} S$ and $\partial_{2} S . \alpha \sim \beta$ implies that if $\gamma \in \mathscr{H}$ intersects with $\alpha$, then $\gamma$ also intersects with $\beta$, and so, $\lambda(\gamma) \in \operatorname{LINK}(a) \cap \operatorname{LINK}(b)$. It follows that $w_{\alpha} \in\langle\operatorname{LINK}(a) \cap \operatorname{LINK}(b)\rangle$. Note that $w_{\partial_{1} S}$ and $w_{\partial_{2} S}$ are in $\langle a, b, \operatorname{LINK}(a) \cap \operatorname{LINK}(b)\rangle$. As in the proof of Lemma 3.4, consider $\delta_{1} \sim \partial_{1} S$ and $\delta_{2} \sim \partial_{2} S$ with the same base point such that $\delta_{1}$ and $\delta_{2}$ transversely intersect $\mathscr{H}$ (Figure 3). We assume that $\delta_{1}$ and $\delta_{2}$ are sufficiently close to $\partial_{1} S$ and $\alpha \cdot \partial_{2} S \cdot \alpha^{-1}$ respectively, so that $w_{\delta_{1}}$ and $w_{\delta_{2}}$ are in $\langle a, b, \operatorname{LINK}(a) \cap \operatorname{LINK}(b)\rangle . \phi\left(\left[\delta_{1}, \delta_{2}\right]\right)=\left[w_{\delta_{1}}, w_{\delta_{2}}\right]=1$, since $\{a, b\} \cup(\operatorname{LINK}(a) \cap \operatorname{LINK}(b))$ induces a complete subgraph in $\Gamma$. This leads to a contradiction again, since $\partial_{1} S \neq \partial_{2} S$ (Lemma 4.3) implies that $\left[\delta_{1}, \delta_{2}\right] \neq 1$ (Lemma 3.1).

Claim 4. If a $b$-arc $\beta$ joins two components in $\partial^{a} S$, then $\beta$ intersects some $\gamma \in \mathcal{H}$ that is not labeled by a vertex in $\operatorname{LINK}(a) \cup\{a\}$.

As in the proof of Claim 3, choose $\beta_{1}$ sufficiently close to $\beta$ such that $\beta_{1} \sim \beta$, $w_{\beta_{1}}=w_{\beta} \in\langle\operatorname{LINK}(b)\rangle$, and $\beta_{1}$ transversely intersects $\mathscr{H}$. Assume that whenever $\gamma \in \mathscr{H}$ and $\beta_{1} \cap \gamma \neq \varnothing, \lambda(\gamma) \in \operatorname{LINK}(a) \cup\{a\}$. This implies $w_{\beta_{1}} \in\langle(\operatorname{LINK}(a) \cup$ $\{a\}) \cap \operatorname{LINK}(b)\rangle=\langle a, \operatorname{LINK}(a) \cap \operatorname{LINK}(b)\rangle$. Let $\partial_{1} S$ and $\partial_{2} S$ be the boundary components joined by $\beta$. $\partial_{i} S \subseteq \partial^{a} S \cap \partial^{b} S$ for $i=1,2$, by the assumption of the claim. This means $w_{\partial_{1} S}, w_{\partial_{2} S} \in\langle a, b, \operatorname{LINK}(a) \cap \operatorname{LINK}(b)\rangle$. As in the proof of Claim 3, [[$\left.\left.\partial_{1} S\right],\left[\beta_{1} \cdot \partial_{2} S \cdot \beta_{1}^{-1}\right]\right]=1$, which is a contradiction. So, there exists $\gamma \in \mathscr{H}$ such that $\beta_{1} \cap \gamma \neq \varnothing$ (hence, $\beta \cap \gamma \neq \varnothing$ ) and $\lambda(\gamma) \notin \operatorname{LINK}(a) \cup\{a\}$.

Now, we recall the notations and the terms from Definition 4.5 and Remark 4.6. For each $\alpha \in \mathcal{A}_{a}, \operatorname{ch}(\alpha)$ denotes the channel of $\alpha$ with respect to the set $\mathcal{A}_{a}$ and $N(\operatorname{ch}(\alpha))$ denotes a sufficiently small closed regular neighborhood of $\operatorname{ch}(\alpha)$ satisfying the conclusion of Lemma 4.8. This implies that $\hat{\alpha}$ is homotopic to the unique component of $\partial N(\operatorname{ch}(\alpha)) \backslash \partial S$ (Lemma 4.8 (1)). 
Claim 5. Let $\alpha$ be an a-arc, and $\hat{\alpha}$ be the unique induced simple closed curve of $\alpha$ with respect to $\mathcal{A}_{a}$. Then $w_{\hat{\alpha}} \in\langle\operatorname{LINK}(a)\rangle$.

From Remark 4.6 that $\hat{\alpha}$ can be written as

$$
\hat{\alpha}=\alpha_{1}^{\prime} \cdot \delta_{1} \cdot \alpha_{2}^{\prime} \cdot \delta_{2} \ldots \alpha_{r}^{\prime}
$$

where for each $i, \alpha_{i}^{\prime}$ is homotopic to an $a$-arc $\alpha_{i}$, and $\delta_{i}$ is an interval on a boundary component of $S$ which is intersecting with the $a$-arcs $\alpha_{i}$ and $\alpha_{i+1}$. Moreover, $\hat{\alpha}$ does not intersect with any $a$-curves or $a$-arcs. It follows that $w_{\hat{\alpha}} \in\langle\operatorname{LINK}(a)\rangle$.

Claim 6. The induced simple closed curve of an a-or b-arc is essential.

Suppose that the induced simple closed curve $\hat{\alpha}$ of an $a$-arc $\alpha$ is null-homotopic. By Lemma 4.8(3), $\partial^{a} S=\partial S$, and any simple closed curve in $\mathscr{H}$ is labeled by a vertex in $\operatorname{LINK}(a)$. This implies that the label of any curve or arc in $\mathscr{H}$ is either $a$ or adjacent to $a$. Hence, $\lambda(\mathscr{H}) \in \operatorname{LINK}(a) \cup\{a\}$, which was excluded. The case for the induced simple closed curve of a $b$-arc is similar, by symmetry.

Claim 7. $\left(\cup \mathcal{A}_{a}\right) \cap\left(\cup \mathcal{C}_{b}\right)=\varnothing$, and $\left(\cup \mathcal{A}_{b}\right) \cap\left(\cup \mathcal{C}_{a}\right)=\varnothing$.

Suppose $\alpha \in \mathcal{A}_{a}$ and $\beta \in \mathscr{C}_{b}$ intersect at $p$. Let $\hat{\alpha}$ be the induced simple closed curve of $\alpha . \hat{\alpha}^{\prime} \sim \hat{\alpha}$ denotes the unique boundary component of $N(\operatorname{ch}(\alpha))$ that is not in $\partial S$ (Lemma $4.8(1))$. $\hat{\alpha}^{\prime} \nsim 0$ by Claim 6. By Claim 5, $w_{\hat{\alpha}^{\prime}} \in\langle\operatorname{LINK}(a)\rangle$. Since $\alpha \cap \beta \neq \varnothing, \hat{\alpha}^{\prime} \cap \beta \neq \varnothing$. Moreover, $w_{\beta} \in\langle\operatorname{LINK}(b)\rangle$. By Claim 1, $\hat{\alpha}^{\prime} \sim \beta^{ \pm 1}$, and so, $i(\alpha, \beta)=i\left(\alpha, \hat{\alpha}^{\prime}\right)=0$. This contradicts to the assumption that $\alpha$ and $\beta$ are minimally intersecting. $\left(\cup \mathcal{A}_{b}\right) \cap\left(\cup \mathcal{C}_{a}\right)=\varnothing$ follows from the symmetry.

Claim 8. Let $\alpha \in \mathcal{A}_{a}$ and $\beta \in \mathcal{A}_{b}$. Denote the induced simple closed curves of $\alpha$ and $\beta$ by $\hat{\alpha}$ and $\hat{\beta}$, respectively. Suppose that either

(i) $\alpha$ and $\beta$ intersect, or

(ii) $\hat{\alpha}$ and $\hat{\beta}$ intersect, and there exists a boundary component which intersects with both $\alpha$ and $\beta$.

Then $\hat{\alpha} \sim \hat{\beta}^{ \pm 1}$ and $N(\operatorname{ch}(\alpha)) \cap \partial S=N(\operatorname{ch}(\beta)) \cap \partial S$. Moreover, there exists a homotopy from $N(\operatorname{ch}(\alpha))$ onto $N(\operatorname{ch}(\beta))$ fixing $N(\operatorname{ch}(\alpha)) \cap \partial S=N(\operatorname{ch}(\beta)) \cap \partial S$.

As in Claim 7, let $\hat{\alpha}^{\prime}$ and $\hat{\beta}^{\prime}$ be the boundary components of $N(\operatorname{ch}(\alpha))$ and $N(\operatorname{ch}(\beta))$, that are not boundary components of $S$, respectively. Assuming (i) or (ii), two essential curves $\hat{\alpha}^{\prime}$ and $\hat{\beta}^{\prime}$ intersect. Here, we have also assumed that $\hat{\alpha}^{\prime}$ and $\hat{\beta}^{\prime}$ are sufficiently close to $\hat{\alpha}$ and $\hat{\beta}$. By Claim $5, w_{\hat{\alpha}^{\prime}} \in\langle\operatorname{LINK}(a)\rangle$ and $w_{\hat{\beta}^{\prime}} \in\langle\operatorname{LINK}(b)\rangle$. From Claim 1, it follows that $\hat{\alpha}^{\prime} \sim \hat{\beta}^{\prime \pm 1}$. By Lemma $4.8(1)$, both $\hat{\alpha}^{\prime}$ and $\hat{\beta}^{\prime}$ are separating simple closed curves on $S$. So either $N(\operatorname{ch}(\alpha)) \sim N(\operatorname{ch}(\beta))$ or $N(\operatorname{ch}(\alpha)) \sim \overline{S \backslash N(\operatorname{ch}(\beta))}$. Suppose that $N(\operatorname{ch}(\alpha)) \sim \overline{S \backslash N(\operatorname{ch}(\beta))}$. Then any boundary component of $S$ contained in $N(\operatorname{ch}(\alpha))$ will not be contained in $N(\operatorname{ch}(\beta))$. 
So no boundary component of $S$ can intersect both $\alpha$ and $\beta$. Since $\alpha \subseteq N(\operatorname{ch}(\alpha))$, $\alpha$ is homotopic into $S \backslash N(\operatorname{ch}(\beta))$ and so, $i(\alpha, \beta)=0$. So neither (i) nor (ii) of the given conditions hold. Therefore, $N(\operatorname{ch}(\alpha)) \sim N(\operatorname{ch}(\beta))$, and the rest of the claim follows immediately.

Claim 9. Let $\alpha$ be an a-arc. Suppose that a $b$-arc $\beta$ joins two boundary components of $S$ that are contained in $N(\operatorname{ch}(\alpha))$. Let $\hat{\alpha}$ and $\hat{\beta}$ denote the induced simple closed curves of $\alpha$ and $\beta$, respectively. Then $\hat{\alpha} \cap \hat{\beta}=\varnothing$.

Suppose that $\beta$ joins $\partial_{1} S$ and $\partial_{2} S$, and $\partial_{1} S \cup \partial_{2} S \subseteq N(\operatorname{ch}(\alpha))$. Assume that $\hat{\alpha} \cap \hat{\beta} \neq \varnothing$. For $i=1$ or $2, \partial_{i} S \subseteq N(\operatorname{ch}(\alpha))$ and so, $\partial_{i} S$ intersects with some $a$-arc. By Claim 4, there exists $\gamma \in \mathscr{H}$ such that $\beta \cap \gamma \neq \varnothing$ and $\lambda(\gamma) \notin \operatorname{LINK}(a) \cup\{a\}$. This implies that $\gamma$ can not intersect any $a$-curve or $a$-arc, and $\gamma \cap \partial^{a} S=\varnothing$. In particular, $\gamma \cap\left(\cup \mathcal{A}_{a}\right)=\varnothing$. By Lemma 4.8 (2), $\gamma \leadsto S \backslash N(\operatorname{ch}(\alpha))$ (Notation 2.2). By choosing a suitable arc in $\operatorname{ch}(\alpha)$, we may assume that $\alpha$ intersects with either $\partial_{1} S$ or $\partial_{2} S$. From Claim 8 (with condition (ii)), $\gamma \leadsto S \backslash N(\operatorname{ch}(\beta))$. So $i(\beta, \gamma)=0$, which is a contradiction.

Claim 10. $\left(\cup \mathcal{A}_{a}\right) \cap\left(\cup \mathcal{A}_{b}\right)=\varnothing$.

Suppose that $\alpha \in \mathcal{A}_{a}$ and $\beta \in \mathcal{A}_{b}$ intersect. By Claim 8 (with condition (i)), $\hat{\alpha} \sim$ $\hat{\beta}^{ \pm 1}$ and $N(\operatorname{ch}(\alpha)) \sim N(\operatorname{ch}(\beta))$. This implies that $N(\operatorname{ch}(\alpha)) \cap \partial S=N(\operatorname{ch}(\beta)) \cap \partial S$, and so, $\beta \subseteq N(\operatorname{ch}(\beta))$ joins two boundary components contained in $N(\operatorname{ch}(\alpha))$. By Claim $9, \hat{\alpha} \cap \hat{\beta}=\varnothing$, which contradicts to the assumption that $\alpha \cap \beta \neq \varnothing$.

Claim 11. $\partial^{a} S \cap \partial^{b} S=\varnothing$.

Suppose that $\partial_{i} S$ intersects with an $a-\operatorname{arc} \alpha$ and a $b$-arc $\beta$. By considering a nearest pair of such arcs on $\partial_{i} S$, we may assume that the induced simple closed curves $\hat{\alpha}$ and $\hat{\beta}$ of $\alpha$ and $\beta$ intersect (Figure 11). By Claim 8 again, $N(\operatorname{ch}(\alpha))=N(\operatorname{ch}(\beta)$ ), and hence as in the proof of Claim 10, $\beta$ joins two boundary components of $N(\operatorname{ch}(\alpha))$. By Claim 9, $\hat{\alpha}$ and $\hat{\beta}$ are disjoint, which is a contradiction. This proves Claim 11.

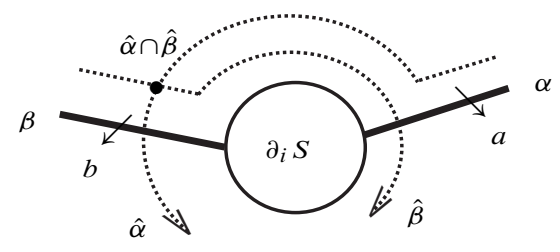

Figure 11. Proof of Claim 11.

Recall $\Gamma^{\prime}=\Gamma \backslash \stackrel{\ominus}{e}$. By Claim 2, 7 and 10, $\alpha$ and $\beta$ are disjoint for any $\alpha \in \lambda^{-1}(a)$ and $\beta \in \lambda^{-1}(b)$. Hence, $(\mathcal{H}, \lambda)$ can be considered as a label-reading pair with the underlying graph $\Gamma^{\prime}$, inducing $\phi^{\prime}: \pi_{1}(S) \rightarrow A\left(\Gamma^{\prime}\right)$. Injectivity of $\phi^{\prime}$ can be seen from 
the following commutative diagram. Here, $A\left(\Gamma^{\prime}\right) \rightarrow A(\Gamma)$ is the natural quotient map, obtained by adding the relator $[a, b]=1$.

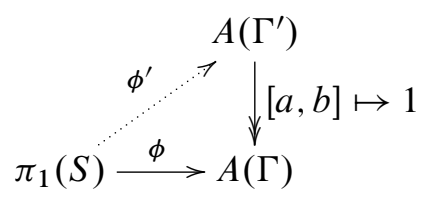

By Claim 11, no boundary components of $S$ intersect with an $a$-arc and a $b$-arc at the same time. So the labels of the arcs intersecting with a fixed boundary component $\partial_{i} S$ are pairwise adjacent not only in $\Gamma$, but also in $\Gamma^{\prime}$. Hence, $\phi^{\prime}$ is a relative embedding, and $\Gamma^{\prime} \notin \mathcal{N}^{\prime}$.

Recall that a graph $\Gamma$ is chordal bipartite if $\Gamma$ does not contain a triangle or an induced cycle of length at least 5 .

Corollary 5.2. Choral bipartite graphs are in $\mathcal{N}^{\prime}$.

Proof. By applying Theorem 3.12 to $K_{0}(=\varnothing)$ amalgamation, one sees that $\mathcal{N}^{\prime}$ is closed under disjoint union. In particular, discrete graphs are in $\mathcal{N}^{\prime}$. Golumbic and Goss proved that by removing bisimplicial edges from a chordal bipartite graph successively, one obtains a discrete graph [13]. By Theorem 5.1, it follows that any chordal bipartite graph is in $\mathcal{N}^{\prime}$.

\section{6. $\mathcal{N}$ and $\mathcal{N}^{\prime}$}

We have shown several properties of the graph class $\mathcal{N}^{\prime}$, which is a subclass of $\mathcal{N}$. In this section, we give a lower bound for $\mathcal{N}^{\prime}$ to illustrate that $\mathcal{N}^{\prime}$ already contains a large number of graphs. Also, we prove that two specific graphs are not in $\mathcal{N}$ (hence not in $\mathcal{N}^{\prime}$ ), providing new examples not covered by the results that we have discussed so far. Finally, we show equivalent formulations of Conjecture 1.1.

Let $\Gamma$ be a graph. Suppose that $B$ is a subset of $V(\Gamma)$ such that the complement graph of the induced subgraph $\Gamma_{B}$ is connected. Recall from [22] that the co-contraction $\overline{\mathrm{CO}}(\Gamma, B)$ of $\Gamma$ relative to $B$ is defined as

$$
\overline{\mathrm{CO}}(\Gamma, B)=\overline{\bar{\Gamma} / \overline{\Gamma_{B}}},
$$

where $\bar{\Gamma} / \overline{\Gamma_{B}}$ denote the graph obtained from $\bar{\Gamma}$ by topologically contracting all the edges in $\overline{\Gamma_{B}}$ onto a vertex and removing loops or multi-edges thus obtained, successively. In [22], it is shown that $A(\overline{\mathrm{CO}}(\Gamma, B))$ embeds into $A(\Gamma)$. Using this, we first prove that $\mathcal{N}^{\prime}$ is closed under co-contraction.

Proposition 6.1. Let $\Gamma$ be a graph that co-contracts onto $\Gamma^{\prime}$. If $\Gamma \in \mathcal{N}^{\prime}$, then $\Gamma^{\prime} \in \mathcal{N}^{\prime}$. 
Proof. Assume that $\Gamma^{\prime} \notin \mathcal{N}^{\prime}$. One can find a compact hyperbolic surface $S$ and a relative embedding $\phi: \pi_{1}(S) \rightarrow A\left(\Gamma^{\prime}\right)$ induced by a normalized label-reading pair $\left(\mathscr{H}^{\prime}, \lambda^{\prime}\right)$. Let $\partial_{1} S, \ldots, \partial_{m} S$ be the boundary components of $S$. Using induction, we have only to consider the case when $\overline{\Gamma^{\prime}}$ is obtained from $\bar{\Gamma}$ by contracting an edge $\{a, b\} \in E(\bar{\Gamma})$ onto a vertex $v \in V\left(\overline{\Gamma^{\prime}}\right)$. By [22], the map $A\left(\Gamma^{\prime}\right) \rightarrow A(\Gamma)$ sending $v$ to $b^{-1} a b$, while sending the other vertices onto themselves, is an embedding. From the definition of a co-contraction, $\operatorname{LINK}(v)=\operatorname{LINK}(a) \cap \operatorname{LINK}(b)$. If $\lambda^{\prime-1}(v)=\varnothing$, then $\phi: \pi_{1}(S) \rightarrow A\left(\Gamma^{\prime} \backslash\{v\}\right)=A(\Gamma \backslash\{a, b\}) \leq A(\Gamma)$, and hence $\Gamma \notin \mathcal{N}^{\prime}$. Now assume $\lambda^{\prime-1}(v) \neq \varnothing$ and choose any $\alpha \in \lambda^{\prime-1}(v)$. First, consider the case when $\alpha$ is an arc. Suppose that $\alpha$ intersects with $\partial_{i} S$. By the definition of a relative embedding, any arc intersecting with $\partial_{i} S$ is labeled by a vertex in $\{v\} \cup \operatorname{LINK}(v)=$ $\{v\} \cup(\operatorname{LINK}(a) \cap \operatorname{LINK}(b))$. Consider the strip $\eta_{\alpha}: I \times[-1,1] \rightarrow S$ containing $\alpha$. We replace $\alpha$ in $\mathscr{H}^{\prime}$ by three homotopic arcs $\alpha_{1}=\eta_{\alpha}(I \times-1), \alpha_{2}=\eta_{\alpha}(I \times 0)$ and $\alpha_{3}=\eta_{\alpha}(I \times 1)$ such that the following hold as in Figure 12 (a).

(i) $\alpha_{1}$ and $\alpha_{3}$ are labeled by $b$.

(ii) $\alpha_{2}$ is labeled by $a$.

(iii) $\alpha_{2}$ and $\alpha_{3}$ have the transverse orientations induced by the homotopies $\alpha_{2} \sim \alpha$ and $\alpha_{3} \sim \alpha$, while $\alpha_{1}$ has the opposite orientations.

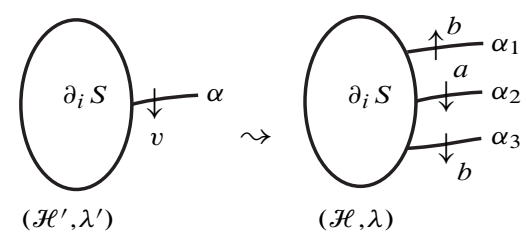

(a)
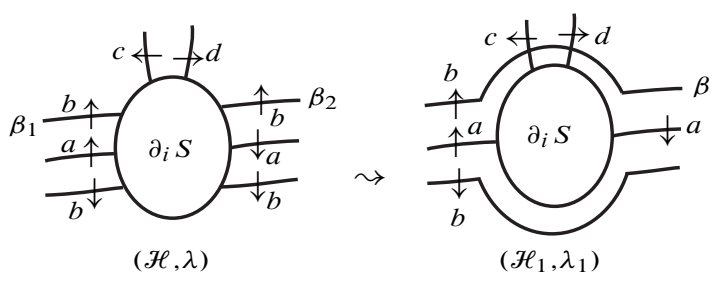

(b)

Figure 12. In (b), $c$ and $d$ belong to $\operatorname{LINK}(a) \cap \operatorname{LINK}(b)$ in $A(\Gamma)$. Hence, $c$-and $d$-arcs are allowed to intersect with the $b$-curve or $b$-arc $\beta$ in $\left(\mathscr{H}_{1}, \lambda_{1}\right)$.

Apply this process for each $v$-arc, and also similarly for each $v$-curve. This results in a new label-reading pair $(\mathscr{H}, \lambda)$ with underlying graph $\Gamma$. The induced label-reading map $\psi: \pi_{1}(S) \rightarrow A(\Gamma)$ is the composition of embeddings $\pi_{1}(S) \hookrightarrow A\left(\Gamma^{\prime}\right) \hookrightarrow A(\Gamma)$. 
In $(\mathscr{H}, \lambda)$, suppose that a $b$-arc intersects with $\partial_{i} S$ for some $i$. Any arcs intersecting with $\partial_{i} S$ are labeled by either $a, b$ or vertices in $\operatorname{LINK}(a) \cap \operatorname{LINK}(b)$. One can pair $b$-arcs intersecting with $\partial_{i} S$, such that for each pair $\left\{\beta_{1}, \beta_{2}\right\}$ :

(i) the transverse orientations of $\beta_{1}$ and $\beta_{2}$ induce opposite orientations on $\partial_{i} S$,

(ii) one of the intervals on $\partial_{i} S \backslash\left(\beta_{1} \cup \beta_{2}\right)$ intersects only with the arcs labeled by $\operatorname{LINK}(a) \cap \operatorname{LINK}(b)$.

Then one can remove intersections between $\partial_{i} S$ and $b$-arcs, without altering the equivalence class of $(\mathscr{H}, \lambda)$ as is illustrated in Figure 12 (b). By applying this process to any $\partial_{i} S$ intersecting with a $b$-arc, we obtain another label-reading pair $\left(\mathscr{H}_{1}, \lambda_{1}\right)$ such that the labels of the arcs intersecting with each boundary component induce a complete subgraph of $\Gamma$. Hence, $\psi$ is a relative embedding, and $\Gamma \notin \mathcal{N}^{\prime}$.

Define $\mathcal{F}$ to be the smallest family of graphs satisfying the following conditions.

(i) $K_{n} \in \mathcal{F}$.

(ii) If $\Gamma_{1}, \Gamma_{2} \in \mathcal{F}$, then $\operatorname{JOIN}\left(\Gamma_{1}, \Gamma_{2}\right) \in \mathcal{F}$.

(iii) If $\Gamma_{1}, \Gamma_{2} \in \mathcal{F}$, and $\Gamma$ is a complete graph amalgamation of $\Gamma_{1}$ and $\Gamma_{2}$, then $\Gamma \in \mathcal{F}$.

(iv) Suppose that $e$ is a bisimplicial edge of a graph $\Gamma$. If $\Gamma \backslash \stackrel{\circ}{e} \in \mathcal{F}$, then $\Gamma \in \mathcal{F}$.

(v) Let $\Gamma \in \mathcal{F}$ and $B \subseteq V(\Gamma)$ such that $\overline{\Gamma_{B}}$ is connected. Then $\overline{\operatorname{CO}}(\Gamma, B) \in \mathcal{F}$.

By the Dirac's result in [8] which was used in the proof of Corollary 3.13, (i) and (iii) imply that chordal graphs are in $\mathcal{F}$. The result of Golumbic and Goss [13] quoted in the proof of Corollary 5.2, along with (iv), implies that any chordal bipartite graphs are in $\mathcal{F}$.

Corollary 6.2. $\mathcal{N}^{\prime}$ contains $\mathcal{F}$.

Proof. Proposition 3.2, Proposition 6.1, Theorem 3.12 and Theorem 5.1 imply that $\mathcal{N}^{\prime}$ is closed under taking a join, taking a co-contraction, amalgamating along a complete subgraph and adding a bisimplicial edge, respectively. Since $\mathcal{F}$ is the smallest of such a graph class, $\mathcal{F} \subseteq \mathcal{N}^{\prime}$.

So, $\mathcal{F}$ provides a lower bound for $\mathcal{N}^{\prime}$. As Corollary 6.2 summarizes techniques introduced in this paper, it seems likely that determining whether $\mathscr{F}=\mathcal{N}^{\prime}$ will require new insights.

Crisp, Sageev and Sapir proposed several reduction moves on underlying graphs of label-reading maps which they successfully used to classify all the graphs in $\mathcal{N}$ with at most 8 vertices [5]. More precisely, they described eight forbidden graphs, and proved that a graph with at most 8 vertices is in $\mathcal{N}$ if and only if the graph does not contain any forbidden graph as an induced subgraph. Their beautiful arguments, especially of finding candidates for kernel elements of label-reading maps, also excluded many graphs with 9 or more vertices from $\mathcal{N}$. However, the question of classifying all the graphs on which right-angled Artin groups contain closed hyperbolic surface groups 
currently seems wide-open. Here, we provide two new examples of graphs that are not in $\mathcal{N}$.

Example 6.3. Crisp, Sageev and Sapir proved that the right-angled Artin group on the graph $P_{1}(8)$ (in their notation) contains a closed hyperbolic surface group [5]; the complement graph of $P_{1}(8)$ is drawn in Figure 13(a). Consider the graphs $\Gamma_{1}$ and $\Gamma_{2}$, whose complements are drawn in Figure $13(\mathrm{~b})$ and (c), respectively. If we topologically contract the edge $\{a, b\}$ in the complement of $\Gamma_{1}$, and remove multiedges thus obtained, then we have the complement graph of $P_{1}(8)$. This means $\Gamma_{1}$ co-contracts onto $P_{1}(8)$; hence, we have an embedding $A\left(P_{1}(8)\right) \hookrightarrow A\left(\Gamma_{1}\right)$ [22]. Similarly, $\overline{\Gamma_{2}}$ contracts onto $\overline{\Gamma_{1}}$ by contracting the edge $\{c, d\}$ onto $a$; so, $A\left(\Gamma_{1}\right)$ embeds into $A\left(\Gamma_{2}\right)$. This implies that $A\left(\Gamma_{1}\right)$ and $A\left(\Gamma_{2}\right)$ contain closed hyperbolic surface groups, since so does $A\left(P_{1}(8)\right)$. One can easily check that $\Gamma_{1}$ and $\Gamma_{2}$ do not contain any forbidden subgraphs considered in [5]. This gives new examples of graphs not in $\mathcal{N}$, hence not in $\mathcal{N}^{\prime}$.

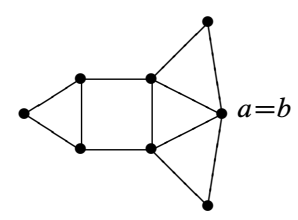

(a) $\overline{P_{1}(8)}$

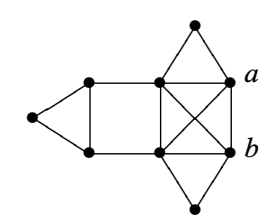

(b) $\overline{\Gamma_{1}}$

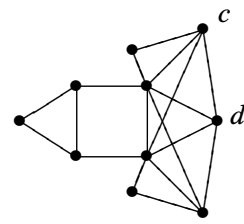

(c) $\overline{\Gamma_{2}}$

Figure 13. The complement graphs of $P_{1}(8), \Gamma_{1}$ and $\Gamma_{2}$.

One of the key obstructions for the question of classifying graphs in $\mathcal{N}$ is Conjecture 1.1. Note that $\mathcal{N}$ is closed under disjoint union and amalgamating along a vertex [21], [5]. We conclude this article by listing equivalent formulations to Conjecture 1.1.

Proposition 6.4. The following are equivalent.

(i) $\mathcal{N}$ is closed under complete graph amalgamation.

(ii) If $\Gamma^{\prime}$ is obtained from $\Gamma$ by removing a simplicial vertex, and $\Gamma^{\prime} \in \mathcal{N}$, then $\Gamma \in \mathcal{N}$.

(iii) $\mathcal{N}^{\prime}=\mathcal{N}$.

Proof. (i) $\Longrightarrow$ (ii) is Obvious, since adding a simplicial vertex to $\Gamma^{\prime}$ is same as amalgamating $\Gamma^{\prime}$ with a complete graph $K_{n}$ along $K_{n-1}$ for some $n$.

For (ii) $\Longrightarrow$ (iii), first note that $\mathcal{N}^{\prime} \subseteq \mathcal{N}$ by definition. To prove $\mathcal{N} \subseteq \mathcal{N}^{\prime}$, suppose that $\Gamma \notin \mathcal{N}^{\prime}$. By Lemma 3.10, $\Gamma^{*}$ is not in $\mathcal{N}$. Note that $\Gamma^{*}$ is obtained from $\Gamma$ by adding independent simplicial vertices to $\Gamma$. Assuming (ii), $\Gamma \notin \mathcal{N}$.

(iii) $\Longrightarrow$ (i) is an immediate from Theorem 3.12 . 


\section{References}

[1] G. Baumslag, On generalised free products. Math.Z. 78(1962), 423-438. Zbl 0104.24402 MR 0140562285

[2] A. J. Casson and S. A. Bleiler, Automorphisms of surfaces after Nielsen and Thurston. London Math. Soc. Stud. Texts 9, Cambridge University Press, Cambridge 1988. Zbl 0649.57008 MR 0964685282

[3] R. Charney, An introduction to right-angled Artin groups. Geom. Dedicata 125 (2007), 141-158. Zbl 1152.20031 MR 2322545277

[4] R. Charney and M. W. Davis, Finite $K(\pi, 1)$ s for Artin groups. In Prospects in topology (Princeton, NJ, 1994), Ann. of Math. Stud. 138, Princeton Univ. Press, Princeton, NJ, 1995, 110-124. Zbl 0930.55006 MR 1368655275

[5] J. Crisp, M. Sageev, and M. Sapir, Surface subgroups of right-angled Artin groups. Internat. J. Algebra Comput. 18 (2008), 443-491. Zbl 05315763 MR 2422070 276, 278, $279,290,304,305$

[6] J. Crisp and B. Wiest, Embeddings of graph braid and surface groups in right-angled Artin groups and braid groups. Algebr. Geom. Topol. 4 (2004), 439-472. Zbl 1057.20028 MR 2077673 275, 277, 279, 280, 281

[7] M. W. Davis and T. Januszkiewicz, Right-angled Artin groups are commensurable with right-angled Coxeter groups. J. Pure Appl. Algebra 153 (2000), 229-235. Zbl 0982.20022 MR 1783167275

[8] G. A. Dirac, On rigid circuit graphs. Abh. Math. Sem. Univ. Hamburg 25 (1961), 71-76. Zbl 0098.14703 MR 0130190 278, 290, 304

[9] C. Droms, Graph groups, coherence, and three-manifolds. J. Algebra 106 (1987), 484-489. Zbl 0692.05035 MR 880971 276, 291

[10] C. Droms, Isomorphisms of graph groups. Proc. Amer. Math. Soc. 100 (1987), 407-408. Zbl 0619.20015 MR 891135275

[11] C. Droms, Subgroups of graph groups. J. Algebra 110 (1987), 519-522. Zbl 0625.20026 MR 910401276

[12] G. Duchamp and J.-Y. Thibon, Simple orderings for free partially commutative groups. Internat. J. Algebra Comput. 2 (1992), 351-355. Zbl 0772.20017 MR 1189240275

[13] M. C. Golumbic and C. F. Goss, Perfect elimination and chordal bipartite graphs. J. Graph Theory 2 (1978), 155-163. Zbl 0411.05060 MR 493395 278, 302, 304

[14] M. C. Golumbic, Algorithmic graph theory and perfect graphs. 2nd ed., Ann. Discrete Math. 57, Elsevier Science B.V., Amsterdam 2004. Zbl 1050.05002 MR 2063679 278, 283

[15] C. M. Gordon, Artin groups, 3-manifolds and coherence. Bol. Soc. Mat. Mexicana (3) 10 (2004), 193-198. Zbl 1100.57001 MR 2199348276

[16] D. Groves and H. Wilton, Conjugacy classes of solutions to equations and inequations over hyperbolic groups. Preprint 2007. arXiv:0710.1892v2 [math.GR] 285

[17] J. Howie, On locally indicable groups. Math. Z. 180 (1982), 445-461. Zbl 0471.20017 MR 667000275 
[18] T. Hsu and D. T. Wise, On linear and residual properties of graph products. Michigan Math. J. 46 (1999), 251-259. Zbl 0962.20016 MR 1704150 275

[19] S. P. Humphries, On representations of Artin groups and the Tits conjecture. J. Algebra 169 (1994), 847-862. Zbl 0822.20041 MR 1302120275

[20] K. H. Kim, L. Makar-Limanov, J. Neggers, and F. W. Roush, Graph algebras. J. Algebra 64 (1980), 46-51. Zbl 0431.05023 MR 575780275

[21] S. Kim, Hyperbolic surface subgroups of right-angled Artin groups and graph products of groups. PhD thesis, Yale University, New Haven 2007. 278, 280, 305

[22] S. Kim, Co-contractions of graphs and right-angled Artin groups. Algebr. Geom. Topol. 8 (2008), 849-868. Zbl 1143.20023 MR 2443098 276, 278, 280, 302, 303, 305

[23] J. Meier and L. VanWyk, The Bieri-Neumann-Strebel invariants for graph groups. Proc. London Math. Soc. (3) 71 (1995), 263-280. Zbl 0835.20037 MR 1337468275

[24] V. Metaftsis and E. Raptis, On the profinite topology of right-angled Artin groups. $J$. Algebra 320 (2008), 1174-1181. Zbl 05354927 MR 2427635276

[25] G. A. Niblo and L. D. Reeves, The geometry of cube complexes and the complexity of their fundamental groups. Topology 37 (1998), 621-633. Zbl 0911.57002 MR 1604899 275

[26] A. Y. Ol'shanskii, Homomorphism diagrams of surface groups. Sibirsk. Mat. Zh. 30 (1989), no. 6, 150-171; English transl. Sib. Math. J. 30 (1989), 961-979. Zbl 0792.20040 MR 1043443280

[27] H. Servatius, C. Droms, and B. Servatius, Surface subgroups of graph groups. Proc. Amer. Math. Soc. 106 (1989), 573-578. Zbl 0677.20023 MR 952322 276, 291

[28] H, Wilton, Solutions to Bestvina \& Feighn's exercises on limit groups. To appear in Geometry and Cohomology in Group Theory (Durham, 4-14 July 2003). arXiv:math/0604137v1 [math.GR] 285

Received September 24, 2008; revised March 30, 2009

S. Kim, Department of Mathematics, The University of Texas at Austin, Austin, TX 78712-0257, U.S.A.

E-mail: shkim@math.utexas.edu 